Yibin Zhang* and Lei Shi

\title{
Concentrating solutions for a planar elliptic problem with large nonlinear exponent and Robin boundary condition
}

https://doi.org/10.1515/anona-2015-0153

Received November 8, 2015; accepted March 9, 2016.

Abstract: Let $\Omega \subset \mathbb{R}^{2}$ be a bounded domain with smooth boundary and $b(x)>0$ a smooth function defined on $\partial \Omega$. We study the following Robin boundary value problem:

$$
\begin{cases}\Delta u+u^{p}=0 & \text { in } \Omega, \\ u>0 & \text { in } \Omega, \\ \frac{\partial u}{\partial v}+\lambda b(x) u=0 & \text { on } \partial \Omega,\end{cases}
$$

where $v$ denotes the exterior unit vector normal to $\partial \Omega, 0<\lambda<+\infty$ and $p>1$ is a large exponent. We construct solutions of this problem which exhibit concentration as $p \rightarrow+\infty$ and simultaneously as $\lambda \rightarrow+\infty$ at points that get close to the boundary, and show that in general the set of solutions of this problem exhibits a richer structure than the problem with Dirichlet boundary condition.

Keywords: Concentrating solutions, large exponent, Robin boundary condition, finite-dimensional reduction

MSC 2010: Primary 35J25; secondary 35B25, 35B38

\section{Introduction}

Let $\Omega \subset \mathbb{R}^{2}$ be a bounded domain with smooth boundary and $b(x)>0$ a smooth function defined on $\partial \Omega$. This paper deals with the analysis of solutions of the Robin boundary value problem

$$
\begin{cases}\Delta u+u^{p}=0 & \text { in } \Omega, \\ u>0 & \text { in } \Omega, \\ \frac{\partial u}{\partial v}+\lambda b(x) u=0 & \text { on } \partial \Omega,\end{cases}
$$

where $v$ denotes the exterior unit vector normal to $\partial \Omega, 0<\lambda<+\infty$ and $p>1$ is a large exponent. Some solutions of (1.1) can be obtained as appropriately scaled extremals of

$$
S_{p}^{\lambda}=\inf _{u \in H^{1}(\Omega) \backslash\{0\}} I_{p}^{\lambda}(u) \quad \text { where } I_{p}^{\lambda}(u)=\frac{\int_{\Omega}|\nabla u|^{2}+\lambda \int_{\partial \Omega} b(x) u^{2}}{\left(\int_{\Omega}|u|^{p+1}\right)^{2 /(p+1)}},
$$

which are guaranteed to exist thanks to the compactness of the Sobolev embedding $H^{1}(\Omega) \rightarrow L^{p+1}(\Omega)$. They are known as least energy solutions of (1.1).

\footnotetext{
*Corresponding Author: Yibin Zhang, College of Sciences, Nanjing Agricultural University, Nanjing 210095, P. R. China, E-mail: yibin10201029@njau.edu.cn

Lei Shi, College of Sciences, Nanjing Agricultural University, Nanjing 210095, P. R. China, E-mail: shileijsxh@163.com
} 
Problem (1.1) has different names depending on the different values of the parameter $\lambda$. It is called Dirichlet if $\lambda=+\infty$, Neumann if $\lambda=0$ and Robin if $0<\lambda<+\infty$. From integration by parts it is trivial to observe that problem (1.1) has no solution when $\lambda=0$. It is also worth pointing out that the Robin boundary condition in (1.1) is particularly interesting in various branches of biological models (see [10,16]). Formally, as $\lambda \rightarrow+\infty$, the Robin boundary condition in (1.1) tends to the Dirichlet boundary condition $\left.u\right|_{\partial \Omega}=0$, and problem (1.1) becomes

$$
\begin{cases}\Delta u+u^{p}=0 & \text { in } \Omega, \\ u>0 & \text { in } \Omega, \\ u=0 & \text { on } \partial \Omega .\end{cases}
$$

Standard variational methods show that problem (1.2) always has a least energy solution $u_{p}$. In [20, 21] it is proved that as $p \rightarrow+\infty$, the solution $u_{p}$ develops one interior peak, namely $u_{p}$ approaches zero except one interior point where it stays bounded and bounded away from zero. Furthermore, up to a subsequence, the renormalized energy $p u_{p}^{p+1}$ concentrates as a Dirac mass around a critical point of the Robin function $H_{\infty}(x, x)$, where $H_{\infty}$ is the regular part of Green's function $G_{\infty}$ of the Dirichlet Laplacian in $\Omega$. Namely, Green's function $G_{\infty}(x, y)$ is the solution of the problem

$$
\begin{cases}-\Delta_{x} G_{\infty}(x, y)=\delta_{y}(x) & \text { in } \Omega, \\ G_{\infty}(x, y)=0 & \text { on } \partial \Omega,\end{cases}
$$

and $H_{\infty}(x, y)$ is the regular part defined as

$$
H_{\infty}(x, y)=G_{\infty}(x, y)-\frac{1}{2 \pi} \log \frac{1}{|x-y|} .
$$

In [1] and [11] Adimurthi and Grossi, and El Mehdi and Grossi, respectively, give a further description of the asymptotic behavior of $u_{p}$ as $p \rightarrow+\infty$, by identifying a limit profile problem of Liouville-type, that is,

$$
\left\{\begin{array}{l}
\Delta u+e^{u}=0 \quad \text { in } \mathbb{R}^{2}, \\
\int_{\mathbb{R}^{2}} e^{u}<+\infty,
\end{array}\right.
$$

and show that $\left\|u_{p}\right\|_{\infty} \rightarrow \sqrt{e}$ as $p \rightarrow+\infty$. On the other hand, problem (1.2) may have solutions with an arbitrarily large number of interior peaks, as shown by Esposito, Musso and Pistoia in [13]. Indeed, they proved that if $\Omega$ is not simply connected, then given any $m \geq 1$ and for $p$ large enough, problem (1.2) has a family of solutions $u_{p}$ which concentrates at $m$ different points of $\Omega$, namely

$$
p u_{p}^{p+1} \rightarrow 8 \pi e \sum_{i=1}^{m} \delta_{\xi_{i}} \quad \text { weakly in the sense of measure in } \bar{\Omega}
$$

as $p \rightarrow+\infty$, and the peaks of these solutions are located near the points $\xi=\left(\xi_{1}, \ldots, \xi_{m}\right) \in \Omega^{m}$ corresponding to a critical point of the function

$$
\varphi_{m}^{\infty}\left(\xi_{1}, \ldots, \xi_{m}\right)=\sum_{j=1}^{m} H_{\infty}\left(\xi_{j}, \xi_{j}\right)+\sum_{j=k} G_{\infty}\left(\xi_{j}, \xi_{k}\right)
$$

More precisely, there is an $m$-tuple $\xi^{p}=\left(\xi_{1, p}, \ldots, \xi_{m, p}\right) \in \Omega^{m}$ converging to $\xi$ such that for any $d>0$, as $p \rightarrow+\infty$,

$$
u_{p} \rightarrow 0 \quad \text { uniformly in } \Omega \backslash \bigcup_{i=1}^{m} B_{d}\left(\xi_{i}^{p}\right) \text { and } \sup _{x \in \Omega \cap B_{d}\left(\xi_{i}^{p}\right)} u_{p}(x) \rightarrow \sqrt{e} .
$$

Additionally, Grossi and Takahashi in [15] prove that when $\Omega$ is convex, problem (1.2) has no solutions that have multiple interior peaks and satisfy property (1.3). Thus, the topological assumption on the domain in [13] is sharp for the construction of multi-peak solutions to problem (1.2).

In contrast, we will see that for any bounded smooth domain, when $\lambda$ and $p$ are large enough, the set of solutions of the so called Robin problem (1.1) is much richer. 
Given $\lambda>0$, let $G_{\lambda}(x, y)$ be Green's function with Robin boundary condition, i.e.,

$$
\begin{cases}-\Delta_{x} G_{\lambda}(x, y)=\delta_{y}(x) & \text { in } \Omega, \\ \frac{\partial G_{\lambda}}{\partial \nu}(x, y)+\lambda b(x) G_{\lambda}(x, y)=0 & \text { on } \partial \Omega,\end{cases}
$$

and let $H_{\lambda}(x, y)$ be its regular part defined as

$$
H_{\lambda}(x, y)=G_{\lambda}(x, y)-\frac{1}{2 \pi} \log \frac{1}{|x-y|} .
$$

As in the Dirichlet problem (1.2), critical points of the Robin function $H_{\lambda}(x, x)$ may play a similar role in determining the location where solutions of problem (1.1) exhibit single-point concentration phenomena. From [7] the Robin function $H_{\lambda}(x, x)$ admits two precise asymptotic formulas, that is, if we set $d(x):=\operatorname{dist}(x, \partial \Omega)$ and introduce the notation $\hat{x}$ as the point on $\partial \Omega$ closest to $x$, then for any $a>1$, there exists $\lambda_{a}$ such that for each $\lambda \geq \lambda_{a}$ and for each $x \in \Omega$ satisfying $a^{-1}<\lambda d(x)<a$, one has that if $b$ is not a constant function,

$$
H_{\lambda}(x, x)=\frac{1}{2 \pi} h_{\lambda}(\lambda d(x), b(\hat{x}))+O\left(\frac{1}{\lambda}\right),
$$

while if $b \equiv 1$,

$$
H_{\lambda}(x, x)=\frac{1}{2 \pi} h_{\lambda}(\lambda d(x), 1)+\frac{1}{2 \pi \lambda} \kappa(\hat{x}) \mathrm{v}(\lambda d(x))+O\left(\frac{1}{\lambda^{1+\alpha}}\right),
$$

where $0<\alpha<1, \kappa(\hat{x})$ is the mean curvature of $\partial \Omega$ at $\hat{x}$

$$
h_{\lambda}(\theta, \tilde{b})=-\log \lambda-\log 2 \theta+2 \int_{0}^{\infty} e^{-t} \log \left(2 \theta+\frac{t}{\tilde{b}}\right) d t
$$

and

$$
\mathrm{v}(\theta)=-\frac{\theta}{2}-\theta \int_{0}^{\infty} e^{-2 \theta s} \frac{1}{(1+s)^{2}} d s .
$$

Moreover, for any $\tilde{b}>0$ the function $h_{\lambda}(\cdot, \tilde{b})$ has a unique minimum $\theta_{\tilde{b}} \in(0,+\infty)$, which is non-degenerate and independent of $\lambda$. Thus, formulas (1.5)-(1.6) suggest that for $\lambda$ and $p$ large enough, problem (1.1) may have solutions which exhibit one or multiple points concentration phenomena in the intermediate region $\lambda d(x)=O(1)$. Our interest here is to find whether these solutions of problem (1.1) exist as $p \rightarrow+\infty$ and simultaneously as $\lambda \rightarrow+\infty$.

The first result we will establish is that when $p$ and $\lambda$ satisfy some restrained growth conditions, for any $m \geq 1$, problem (1.1) possesses at least two solutions which concentrate at $m$ different points with distance to the boundary uniformly approaching zero. Meanwhile, the location of such $m$ points is related to critical points of the function

$$
\varphi_{m}^{\lambda}\left(\xi_{1}, \ldots, \xi_{m}\right)=\sum_{j=1}^{m} H_{\lambda}\left(\xi_{j}, \xi_{j}\right)+\sum_{j=k} G_{\lambda}\left(\xi_{j}, \xi_{k}\right) .
$$

Theorem 1.1. Let $m \geq 1$ be an integer. There exist $p_{m}>0$ and $\lambda_{m}>0$ such that for any $p>p_{m}$ and $\lambda>\lambda_{m}$ satisfying $\frac{\log \lambda}{p}=o(1)$ and $\frac{\lambda}{p^{2} \log \lambda}=O(1)$, there are at least two solutions $u_{p, \lambda}$ for problem (1.1) with $m$ different concentration points $\xi_{1, p, \lambda}, \ldots, \xi_{m, p, \lambda}$ located at distance $O(1 / \lambda)$ from $\partial \Omega$. More precisely,

$$
u_{p, \lambda}(x)=\sum_{j=1}^{m} \frac{1}{y \mu_{j}^{2 /(p-1)}}\left[\log \frac{1}{\left(\delta_{j}^{2}+\left|x-\xi_{j, p, \lambda}\right|^{2}\right)^{2}}+8 \pi H_{\lambda}\left(x, \xi_{j, p, \lambda}\right)\right]+O\left(\frac{1}{p}\right),
$$

where the parameters $y, \delta_{j}$ and $\mu_{j}$ satisfy

$$
y=p^{p /(p-1)} \rho^{2 /(p-1)}, \quad \delta_{j}=\mu_{j} \rho, \quad \rho=\frac{1}{\lambda} e^{-p / 4}, \quad \frac{1}{C}<\mu_{j}<C
$$


for some $C>0$, and $\xi_{j, p, \lambda} \in \Omega$ satisfies

$$
\left|\lambda d\left(\xi_{j, p, \lambda}\right)-\theta_{b\left(\hat{\xi}_{j, p, \lambda}\right)}\right|=O\left(\lambda^{-1 / 2}\right) \quad \text { as } \lambda \rightarrow+\infty
$$

and

$$
\lim _{p \rightarrow+\infty} \nabla \varphi_{m}^{\lambda}\left(\xi_{1, p, \lambda}, \ldots, \xi_{m, p, \lambda}\right)=0 \quad \text { uniformly for all large } \lambda,
$$

where $\varphi_{m}^{\lambda}$ is defined in (1.9). In particular, for any $\varepsilon \in(0,1)$, as $\lambda \rightarrow+\infty$,

$$
\begin{aligned}
p u_{p, \lambda}^{p+1}-8 \pi e \sum_{j=1}^{m} \delta_{\xi_{j, p, \lambda}} & \rightarrow 0 \quad \text { weakly in the sense of measure in } \bar{\Omega}, \\
u_{p, \lambda} \rightarrow 0 & \text { uniformly in } \bar{\Omega} \backslash \bigcup_{j=1}^{m} B_{\varepsilon d\left(\xi_{j, p, \lambda}\right)}\left(\xi_{j, p, \lambda}\right),
\end{aligned}
$$

and

$$
\sup _{B_{\varepsilon d\left(\xi_{j, p, \lambda}\right)}\left(\xi_{j, p, \lambda}\right)} u_{p, \lambda} \rightarrow \sqrt{e} .
$$

Note that as in the Dirichlet problem (1.2), when $\Omega$ is not simple connected, one can find that given any $m \geq 1$, for any fixed $\lambda$ large, problem (1.1) has a third solution $u_{p, \lambda}$ with $m$ different peak points $\xi_{1, p, \lambda}, \ldots, \xi_{m, p, \lambda}$ uniformly away from the boundary and each other as $p \rightarrow+\infty$. We will not provide the construction of this solution because it can be easily realized using the same approach as in [13]. Besides, under a faster restrained growth condition of $p$ and $\lambda$, we also obtain the case $m=1$ of Theorem 1.1 and find solutions with a concentration point near the boundary.

Theorem 1.2. There exist $p_{0}>0$ and $\lambda_{0}>0$ such that for any $p>p_{0}$ and $\lambda>\lambda_{0}$ satisfying $\frac{1}{p} \log \lambda=o(1)$, problem (1.1) has at least two solutions $u_{p, \lambda}$ with a concentration point $\xi_{p, \lambda}$ such that

$$
\left|\lambda d\left(\xi_{p, \lambda}\right)-\theta_{b\left(\hat{\xi}_{p, \lambda}\right)}\right|=O\left(\lambda^{-1 / 2}\right) \quad \text { as } \lambda \rightarrow+\infty .
$$

The following theorems deal with the case $b \equiv 1$ and the case where $b$ is not a constant function, respectively.

Theorem 1.3. Assume $b \equiv 1$. If $x_{0} \in \partial \Omega$ is a non-degenerate critical point of $\kappa$, then for each $\beta \in(0,1)$, there exist $p_{0}>0$ and $\lambda_{0}>0$ such that for any $p>p_{0}$ and $\lambda>\lambda_{0}$ satisfying $\frac{1}{p} \lambda^{1+\beta}=o(1)$, problem (1.1) has a solution $u_{p, \lambda}$ with a concentration point $\xi_{p, \lambda}$ such that

$$
\left|\lambda d\left(\xi_{p, \lambda}\right)-\theta_{b}\right|=O\left(\lambda^{-1 / 2}\right) \text { and }\left|\hat{\xi}_{p, \lambda}-x_{0}\right|=O\left(\lambda^{-\beta}\right) \text { as } \lambda \rightarrow+\infty .
$$

Theorem 1.4. Let $x_{0} \in \partial \Omega$ be a non-degenerate critical point of $b$. Then, for each $\beta \in(0,1)$, there exist $p_{0}>0$ and $\lambda_{0}>0$ such that for any $p>p_{0}$ and $\lambda>\lambda_{0}$ satisfying $\frac{1}{p}\left(\lambda^{(1+\beta) / 3}+\lambda^{\beta}\right)=o(1)$, problem (1.1) has a solution $u_{p, \lambda}$ with a concentration point $\xi_{p, \lambda}$ such that

$$
\left|\lambda d\left(\xi_{p, \lambda}\right)-\theta_{b\left(\hat{\xi}_{p, \lambda}\right)}\right|=O\left(\lambda^{-1 / 2}\right) \text { and }\left|\hat{\xi}_{p, \lambda}-x_{0}\right|=O\left(\lambda^{-\beta}\right) \text { as } \lambda \rightarrow+\infty .
$$

Let us remark the interesting analogy between all above results and those known for the Liouville equation with Robin boundary condition

$$
\begin{cases}\Delta u+\varepsilon^{2} e^{u}=0 & \text { in } \Omega, \\ \frac{\partial u}{\partial v}+\lambda b(x) u=0 & \text { on } \partial \Omega,\end{cases}
$$

where $\Omega \subset \mathbb{R}^{2}$ is a bounded smooth domain, $\varepsilon>0$ is small but $\lambda>0$ is large, and $b(x)>0$ is a smooth function defined on $\partial \Omega$. For $b(x) \equiv 1$, Davila and Topp in [8] construct solutions of problem (1.10) which exhibit concentration phenomena as $\varepsilon \rightarrow 0$ and simultaneously as $\lambda \rightarrow+\infty$ at one or multiple points that get close to 
the boundary. More precisely, they prove that if $\varepsilon$ and $\lambda$ satisfy some restrained condition, then for any $m \geq 1$, problem (1.10) has at least two solutions with $m$ different concentration points located at distance $O(1 / \lambda)$ from $\partial \Omega$, while if $\varepsilon$ and $\lambda$ satisfy a weaker restrained condition so that $\lambda$ has a faster growth, problem (1.10) has at least two solutions with a concentration point near the boundary. Additionally, when $x_{0}$ is a non-degenerate critical point of the mean curvature function $\kappa$ on $\partial \Omega$, they find that under a certain restrained condition of $p$ and $\lambda$, problem (1.10) has a solution with a concentration point located at distance $O(1 / \lambda)$ from $x_{0}$.

Finally, it is necessary to compare Theorem 1.2 with the results given in [3], which is concerned with the following singularly perturbed elliptic problem with Robin boundary condition:

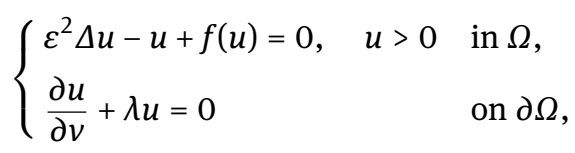

where $\Omega \subset \mathbb{R}^{N}(N \geq 2)$ is a bounded smooth domain, $\varepsilon>0$ is a small constant, $0<\lambda<+\infty, f(u)=u^{p}-a u^{q}$ with $a \geq 0$, and $1<q<p<\frac{N+2}{N-2}$ if $N \geq 3$ whereas $1<q<p<+\infty$ if $N=2$. The cases $\lambda=0$ (Neumann problem) and $\lambda=+\infty$ (Dirichlet problem) have been studied, respectively, in [17, 18] and [19], proving that the least energy solution of problem (1.11) with Dirichlet boundary condition concentrates a point that maximizes the distance to the boundary, while Neumann concentration takes place at a point on the boundary. In [3] it is proved that for fixed small $\varepsilon$, as $\lambda$ moves from 0 to $+\infty$, the asymptotic behavior of the least energy solution of problem (1.11) changes dramatically. It will be like in the case of Neumann boundary condition if $\lambda<\lambda_{\star} / \varepsilon$ and like in the case of Dirichlet boundary condition if $\lambda>\lambda_{\star} / \varepsilon$, where $\lambda_{\star}$ is the borderline number associated to the corresponding problem on the half space. Thus, $\lambda \sim 1 / \varepsilon$ represents a dramatic change in asymptotic behavior of the least energy solution of problem (1.11). Our results suggest that for least energy solutions of problem (1.1), the critical range of $\lambda$ may be $\log \lambda \sim p$.

The general strategy for proving our main results relies on the very well-known Lyapunov-Schmidt reduction, which has been used in many papers, for example, in $[8,9,12,13]$. The paper is organized as follows. In Section 2 we exactly describe the ansatz for the solution of problem (1.1) and estimate the error. Then, we rewrite problem (1.1) in terms of a linearized operator for which a solvability theory, subject to suitable orthogonality conditions, is performed through solving a linearized problem in Section 3. In Section 4 we solve an auxiliary nonlinear problem. In Section 5 we reduce (1.1) to a finite system. In the last section, we give the proof of Theorems 1.1-1.4.

In this paper, the symbol $C$ denotes always a generic positive constant independent of $p$ and $\lambda$, which could be changed from one line to another. The symbols $O(t)$ (respectively $o(t)$ ) denote quantities for which $\frac{O(t)}{|t|}$ stays bounded (respectively, $\frac{o(t)}{|t|}$ tends to zero) as the parameter $t$ goes to zero. In particular, we will often use the notations $O(1)$ (respectively, $o(1)$ ), which represents a quantity that remains uniformly bounded (respectively, that tends to zero) as $p \rightarrow+\infty$ and simultaneously as $\lambda \rightarrow+\infty$.

\section{A first approximation of the solution}

In this section we will provide an ansatz for solutions of problem (1.1). A key ingredient to describe an approximate solution of problem (1.1) is given by the standard bubble:

$$
U_{\delta, \xi}(x)=\log \frac{8 \delta^{2}}{\left(\delta^{2}+|x-\xi|^{2}\right)^{2}}, \quad \delta>0, \xi \in \mathbb{R}^{2} .
$$

It is well known (see [5]) that those are all the solutions of the following Liouville-type equation:

$$
\left\{\begin{array}{l}
\Delta u+e^{u}=0 \quad \text { in } \mathbb{R}^{2}, \\
\int_{\mathbb{R}^{2}} e^{u}<+\infty .
\end{array}\right.
$$

The configuration space we try to look for $m$ concentration points $\xi=\left(\xi_{1}, \ldots, \xi_{m}\right)$ is the following:

$$
\mathcal{O}_{\varepsilon}:=\left\{\xi=\left(\xi_{1}, \ldots, \xi_{m}\right) \in \Omega^{m}:\left|\xi_{j}-\xi_{k}\right| \geq \varepsilon,\left|\lambda d\left(\xi_{j}\right)-\theta_{b\left(\hat{\xi}_{j}\right)}\right| \leq \frac{1}{\varepsilon \lambda^{1 / 2}}, j, k=1, \ldots, m, j=k\right\},
$$


where $\varepsilon>0$ is a sufficiently small but fixed number. Note that by the choice of $\xi_{j}$, if $\xi=\left(\xi_{1}, \ldots, \xi_{m}\right) \in \mathcal{O}_{\varepsilon}$, then we have

$$
d\left(\xi_{j}\right)=\operatorname{dist}\left(\xi_{j}, \partial \Omega\right)=O\left(\frac{1}{\lambda}\right) \text { and } \theta_{b\left(\hat{\xi}_{j}\right)}=O(1) \quad \text { for all } j=1, \ldots, m .
$$

In what remains of this paper, we will always assume the following restrained growth condition:

$$
\frac{1}{p} \log \lambda \rightarrow 0 \text { as } p \rightarrow+\infty \text { and simultaneously as } \lambda \rightarrow+\infty .
$$

Also, for each $j=1, \ldots, m$, we set

$$
y=p^{p /(p-1)} \rho^{2 /(p-1)}, \quad \delta_{j}=\mu_{j} \rho, \quad \rho=\frac{1}{\lambda} e^{-p / 4},
$$

where the choice of $\mu_{j}$ will be determined later. Define now

$$
U_{j}(x)=\frac{1}{y \mu_{j}^{2 /(p-1)}}\left[U_{\delta_{j}, \xi_{j}}(x)+\frac{1}{p} \omega_{1}\left(\frac{x-\xi_{j}}{\delta_{j}}\right)+\frac{1}{p^{2}} \omega_{2}\left(\frac{x-\xi_{j}}{\delta_{j}}\right)\right] .
$$

Here, $\omega_{i}, i=1,2$, are radial solutions of

$$
\Delta \omega_{i}+\frac{8}{\left(1+|y|^{2}\right)^{2}} \omega_{i}=\frac{8}{\left(1+|y|^{2}\right)^{2}} f_{i}(y) \text { in } \mathbb{R}^{2}
$$

with

$$
f_{1}=\frac{1}{2} U_{1,0}^{2}, \quad f_{2}=\omega_{1} U_{1,0}-\frac{1}{3} U_{1,0}^{3}-\frac{1}{2} \omega_{1}^{2}-\frac{1}{8} U_{1,0}^{4}+\frac{1}{2} \omega_{1} U_{1,0}^{2},
$$

having the asymptotic properties

$$
\left\{\begin{array}{cl}
\omega_{i}(y)=C_{i} \log |y|+O\left(\frac{1}{|y|}\right) & \text { as }|y| \rightarrow+\infty, \\
\nabla \omega_{i}(y)=C_{i} \cdot \frac{y}{1+|y|^{2}}+O\left(\frac{1}{1+|y|^{2}}\right) & \text { for all } y \in \mathbb{R}^{2},
\end{array}\right.
$$

where

$$
C_{i}=8 \int_{0}^{\infty} t \frac{t^{2}-1}{\left(t^{2}+1\right)^{3}} f_{i}(t) d t
$$

In particular,

$$
\begin{aligned}
\omega_{1}(y)=\frac{1}{2} & U_{1,0}^{2}(y)+6 \log \left(|y|^{2}+1\right)+\frac{2 \log 8-10}{|y|^{2}+1}+\frac{|y|^{2}-1}{|y|^{2}+1} \\
& \times\left\{-\frac{1}{2} \log ^{2} 8+2 \log ^{2}\left(|y|^{2}+1\right)+4 \int_{|y|^{2}}^{\infty} \frac{d s}{s+1} \log \frac{s+1}{s}-8 \log |y| \log \left(|y|^{2}+1\right)\right\}
\end{aligned}
$$

and (see $[4,13])$

$$
C_{1}=12-4 \log 8 .
$$

Our ansatz for a solution of problem (1.1) is

$$
U_{\xi}(x)=\sum_{j=1}^{m}\left[U_{j}(x)+H_{j}(x)\right],
$$

where $H_{j}$ is a correction term defined as the solution of the following problem:

$$
\begin{cases}-\Delta H_{j}=0 & \text { in } \Omega, \\ \frac{\partial H_{j}}{\partial v}+\lambda b(x) H_{j}=-\frac{\partial U_{j}}{\partial v}-\lambda b(x) U_{j} & \text { on } \partial \Omega .\end{cases}
$$


Lemma 2.1. For any points $\xi=\left(\xi_{1}, \ldots, \xi_{m}\right) \in \mathcal{O}_{\varepsilon}$,

$$
H_{j}(x)=\frac{1}{y \mu_{j}^{2 /(p-1)}}\left[\left(1-\frac{C_{1}}{4 p}-\frac{C_{2}}{4 p^{2}}\right) 8 \pi H_{\lambda}\left(x, \xi_{j}\right)-\log \left(8 \mu_{j}^{2} \rho^{2}\right)+\left(\frac{C_{1}}{p}+\frac{C_{2}}{p^{2}}\right) \log \left(\mu_{j} \rho\right)+O\left(\frac{\mu_{j} \lambda \rho}{p}\right)\right]
$$

uniformly in $C(\bar{\Omega})$ and in $C_{\mathrm{loc}}^{2}(\Omega)$ as $p$ and $\lambda$ go to $+\infty$ and satisfy (2.4), where $H_{\lambda}$ is the regular part of Green's function defined in (1.4).

Proof. First, on the boundary, by (2.1) and (2.9), we have

$$
\begin{aligned}
\frac{\partial H_{j}}{\partial \nu}+\lambda b(x) H_{j}=- & \frac{1}{y \mu_{j}^{2 /(p-1)}}\left\{\left(-4+\frac{C_{1}}{p}+\frac{C_{2}}{p^{2}}\right) \frac{\left(x-\xi_{j}\right) \cdot v(x)}{\mu_{j}^{2} \rho^{2}+\left|x-\xi_{j}\right|^{2}}\right. \\
& +\lambda b(x)\left[\log \frac{1}{\left(\mu_{j}^{2} \rho^{2}+\left|x-\xi_{j}\right|^{2}\right.}+\left(\frac{C_{1}}{p}+\frac{C_{2}}{p^{2}}\right) \log \left|x-\xi_{j}\right|\right] \\
& \left.+\lambda b(x)\left[\log \left(8 \mu_{j}^{2} \rho^{2}\right)-\left(\frac{C_{1}}{p}+\frac{C_{2}}{p^{2}}\right) \log \left(\mu_{j} \rho\right)\right]+\frac{1}{p} O\left(\frac{\mu_{j} \rho}{\left|x-\xi_{j}\right|^{2}}+\frac{\lambda \mu_{j} \rho}{\left|x-\xi_{j}\right|}\right)\right\} .
\end{aligned}
$$

The regular part of Green's function with Robin boundary condition $H_{\lambda}\left(x, \xi_{j}\right)$ satisfies

$$
\begin{cases}-\Delta H_{\lambda}\left(x, \xi_{j}\right)=0 & \text { in } \Omega, \\ \frac{\partial H_{\lambda}\left(x, \xi_{j}\right)}{\partial v}+\lambda b(x) H_{\lambda}\left(x, \xi_{j}\right)=\frac{1}{2 \pi} \frac{\left(x-\xi_{j}\right) \cdot v(x)}{\left|x-\xi_{j}\right|^{2}}-\frac{1}{2 \pi} \lambda b(x) \log \frac{1}{\left|x-\xi_{j}\right|} & \text { on } \partial \Omega .\end{cases}
$$

So, if we set

$$
\widetilde{H}_{j}(x)=y \mu_{j}^{2 /(p-1)} H_{j}(x)-\left[\left(1-\frac{C_{1}}{4 p}-\frac{C_{2}}{4 p^{2}}\right) 8 \pi H_{\lambda}\left(x, \xi_{j}\right)-\log \left(8 \mu_{j}^{2} \rho^{2}\right)+\left(\frac{C_{1}}{p}+\frac{C_{2}}{p^{2}}\right) \log \left(\mu_{j} \rho\right)\right],
$$

then $\widetilde{H}_{j}(x)$ satisfies $-\Delta \widetilde{H}_{j}=0$ in $\Omega$, and

$$
\frac{\partial \widetilde{H}_{j}}{\partial \nu}+\lambda b(x) \widetilde{H}_{j}=-\frac{4 \mu_{j}^{2} \rho^{2}}{\mu_{j}^{2} \rho^{2}+\left|x-\xi_{j}\right|^{2}} \frac{\left(x-\xi_{j}\right) \cdot v(x)}{\left|x-\xi_{j}\right|^{2}}-\lambda b(x) \log \frac{\left|x-\xi_{j}\right|^{4}}{\left(\mu_{j}^{2} \rho^{2}+\left|x-\xi_{j}\right|^{2}\right)^{2}}+\frac{1}{p} O\left(\frac{\mu_{j} \rho}{\left|x-\xi_{j}\right|^{2}}+\frac{\lambda \mu_{j} \rho}{\left|x-\xi_{j}\right|}\right)
$$

on $\partial \Omega$. From the maximum principle with Robin boundary condition (see [7, Lemma 2.6]), we deduce

$$
\max _{\bar{\Omega}}\left|\widetilde{H}_{j}(x)\right|+\max _{\bar{\Omega}}\left|d(x) \nabla \widetilde{H}_{j}(x)\right| \leq \frac{C}{\lambda}\left\|\frac{\partial \widetilde{H}_{j}}{\partial v}+\lambda \widetilde{H}_{j}\right\|_{L^{\infty}(\partial \Omega)}=\frac{1}{p} O\left(\frac{\mu_{j} \rho}{\lambda d\left(\xi_{j}\right)^{2}}+\frac{\mu_{j} \rho}{d\left(\xi_{j}\right)}\right)=O\left(\frac{\mu_{j} \lambda \rho}{p}\right) .
$$

Thus, using the interior estimate of the derivative of a harmonic function (see [14, Theorem 2.10]), we get

$$
\max _{K}\left|D^{\alpha} \widetilde{H}_{j}(x)\right| \leq\left(\frac{2|\alpha|}{\operatorname{dist}(K, \partial \Omega)}\right)^{|\alpha|} \max _{\bar{\Omega}}\left|\widetilde{H}_{j}(x)\right|=O\left(\frac{\mu_{j} \lambda \rho}{p}\right)
$$

for any compact subset $K$ of $\Omega$ and any multi-index $\alpha$ with $|\alpha| \leq 2$. This derives estimate (2.14) uniformly in $C(\bar{\Omega})$ and in $C_{\text {loc }}^{2}(\Omega)$ for any sufficiently large $p$ and $\lambda$ satisfying (2.4).

From Lemma 2.1, away from the points $\xi_{j}$, namely $\left|x-\xi_{j}\right| \geq \varepsilon d\left(\xi_{j}\right)$ for any $j=1, \ldots, m$, one has

$$
U_{\xi}(x)=\sum_{j=1}^{m} \frac{1}{y \mu_{j}^{2 /(p-1)}}\left[\left(1-\frac{C_{1}}{4 p}-\frac{C_{2}}{4 p^{2}}\right) 8 \pi G_{\lambda}\left(x, \xi_{j}\right)+\frac{1}{p} O\left(\mu_{j} \lambda \rho\right)\right] .
$$

While for $\left|x-\xi_{j}\right| \leq \varepsilon d\left(\xi_{j}\right)$ with some $j$, if we write $x=\xi_{j}+\delta_{j} y$, then, using (2.2), (2.5), (2.6), (2.12), (2.14), (2.16) and the fact that $\left|\nabla_{x} H_{\lambda}\left(x, \xi_{j}\right)\right|=O(\lambda)$ uniformly holds in the intermediate region $\lambda d(x)=O(1)$ (see [7]), we 
deduce

$$
\begin{aligned}
U_{\xi}(x)= & \frac{1}{y \mu_{j}^{2 /(p-1)}}\left\{U_{\delta_{j}, \xi_{j}}(x)+\frac{1}{p} \omega_{1}\left(\frac{x-\xi_{j}}{\delta_{j}}\right)+\frac{1}{p^{2}} \omega_{2}\left(\frac{x-\xi_{j}}{\delta_{j}}\right)+\left(1-\frac{C_{1}}{4 p}-\frac{C_{2}}{4 p^{2}}\right) 8 \pi H_{\lambda}\left(x, \xi_{j}\right)\right. \\
& \left.-\log \left(8 \mu_{j}^{2} \rho^{2}\right)+\left(\frac{C_{1}}{p}+\frac{C_{2}}{p^{2}}\right) \log \left(\mu_{j} \rho\right)+\frac{1}{p} O\left(\mu_{j} \lambda \rho\right)\right\} \\
& +\sum_{k=j} \frac{1}{y \mu_{k}^{2 /(p-1)}}\left[\left(1-\frac{C_{1}}{4 p}-\frac{C_{2}}{4 p^{2}}\right) 8 \pi G_{\lambda}\left(x, \xi_{k}\right)+\frac{1}{p} O\left(\mu_{k} \lambda \rho\right)\right] \\
= & \frac{1}{y \mu_{j}^{2 /(p-1)}}\left\{U_{1,0}(y)-\log \left(\mu_{j}^{2} \rho^{2}\right)+\frac{1}{p} \omega_{1}(y)+\frac{1}{p^{2}} \omega_{2}(y)+\left(1-\frac{C_{1}}{4 p}-\frac{C_{2}}{4 p^{2}}\right) 8 \pi H_{\lambda}\left(\xi_{j}, \xi_{j}\right)\right. \\
& -\log \left(8 \mu_{j}^{2} \rho^{2}\right)+\left(\frac{C_{1}}{p}+\frac{C_{2}}{p^{2}}\right) \log \left(\mu_{j} \rho\right)+O\left(\lambda \delta_{j}|y|\right)+\frac{1}{p} O\left(\mu_{j} \lambda \rho\right) \\
& \left.+\sum_{k=j} \frac{\mu_{j}^{2 /(p-1)}}{\mu_{k}^{2 /(p-1)}}\left[\left(1-\frac{C_{1}}{4 p}-\frac{C_{2}}{4 p^{2}}\right) 8 \pi G_{\lambda}\left(\xi_{j}, \xi_{k}\right)+O\left(\lambda \delta_{j}|y|\right)+\frac{1}{p} O\left(\mu_{k} \lambda \rho\right)\right]\right\} \\
= & \frac{1}{y \mu_{j}^{2 /(p-1)}}\left\{p+U_{1,0}(y)+\frac{1}{p} \omega_{1}(y)+\frac{1}{p^{2}} \omega_{2}(y)+\left(1-\frac{C_{1}}{4 p}-\frac{C_{2}}{4 p^{2}}\right) 8 \pi H_{\lambda}\left(\xi_{j}, \xi_{j}\right)\right. \\
& \quad-\log \left(8 \mu_{j}^{4}\right)+4 \log \lambda+\left(\frac{C_{1}}{p}+\frac{C_{2}}{p^{2}}\right) \log \left(\mu_{j} \rho\right)+O\left(\lambda \mu_{j} \rho|y|\right)+\frac{1}{p} O\left(\mu_{j} \lambda \rho\right) \\
& \left.+\sum_{k=j} \frac{\mu_{j}^{2 /(p-1)}}{\mu_{k}^{2 /(p-1)}}\left[\left(1-\frac{C_{1}}{4 p}-\frac{C_{2}}{4 p^{2}}\right) 8 \pi G_{\lambda}\left(\xi_{j}, \xi_{k}\right)+O\left(\lambda \mu_{j} \rho|y|\right)+\frac{1}{p} O\left(\mu_{k} \lambda \rho\right)\right]\right\} .
\end{aligned}
$$

We now choose the parameters $\mu_{j}$. We assume they are defined by the relation

$$
\begin{aligned}
\log \left(8 \mu_{j}^{4}\right)= & 4 \log \lambda+\left(1-\frac{C_{1}}{4 p}-\frac{C_{2}}{4 p^{2}}\right) 8 \pi H_{\lambda}\left(\xi_{j}, \xi_{j}\right)+\left(\frac{C_{1}}{p}+\frac{C_{2}}{p^{2}}\right) \log \left(\mu_{j} \frac{1}{\lambda} e^{-p / 4}\right) \\
& +\sum_{k=j} \frac{\mu_{j}^{2 /(p-1)}}{\mu_{k}^{2 /(p-1)}}\left(1-\frac{C_{1}}{4 p}-\frac{C_{2}}{4 p^{2}}\right) 8 \pi G_{\lambda}\left(\xi_{j}, \xi_{k}\right) .
\end{aligned}
$$

Taking into account the explicit expression (2.11) of the constant $C_{1}$, we observe that as $p$ tends to $+\infty, \mu_{j}$ bifurcates from the value

$$
\bar{\mu}_{j}=\lambda e^{-3 / 4} e^{2 \pi H_{\lambda}\left(\xi_{j}, \xi_{j}\right)+2 \pi \sum_{k=j} G_{\lambda}\left(\xi_{j}, \xi_{k}\right)}
$$

solution of the equation

$$
\log \left(8 \mu_{j}^{4}\right)=4 \log \lambda+8 \pi H_{\lambda}\left(\xi_{j}, \xi_{j}\right)-\frac{1}{4} C_{1}+8 \pi \sum_{k=j} G_{\lambda}\left(\xi_{j}, \xi_{k}\right)
$$

Thus, by (2.4), $\mu_{j}$ is a perturbation of order $\frac{\log \lambda}{p}$ of the value $\bar{\mu}_{j}$, namely

$$
\mu_{j}=\lambda e^{-3 / 4} e^{2 \pi H_{\lambda}\left(\xi_{j}, \xi_{j}\right)+2 \pi \sum_{k=j} G_{\lambda}\left(\xi_{j}, \xi_{k}\right)}\left[1+O\left(\frac{\log \lambda}{p}\right)\right] .
$$

Moreover, from (1.5)-(1.8) and (2.2), there exists a constant $C>0$ independent of $p, \lambda$ and $j$ such that for any sufficiently large $p$ and $\lambda$ satisfying (2.4),

$$
\frac{1}{C}<\mu_{j}<C
$$

and

$$
\left|D_{\xi} \log \mu_{j}\right|<C \sqrt{\lambda}
$$

where the second inequality is due to the fact that if $x \in \Omega$ satisfies $\left|\lambda \operatorname{dist}(x, \partial \Omega)-\theta_{b(\hat{x})}\right|=O\left(\lambda^{-1 / 2}\right)$, then

$$
\left|\nabla_{x} H_{\lambda}(x, x)\right|=O(\sqrt{\lambda}) .
$$


From this choice of the parameters $\mu_{j}$, we deduce that for $\left|x-\xi_{j}\right|=\delta_{j}|y| \leq \varepsilon d\left(\xi_{j}\right)$,

$$
U_{\xi}(x)=\frac{1}{y \mu_{j}^{2 /(p-1)}}\left[p+U_{1,0}(y)+\frac{1}{p} \omega_{1}(y)+\frac{1}{p^{2}} \omega_{2}(y)+O(\lambda \rho|y|)+\frac{1}{p} O(\lambda \rho)\right] .
$$

Remark 2.2. Let us remark that $U_{\xi}$ is a positive uniformly bounded function. Observe that for $|y| \leq \varepsilon d\left(\xi_{j}\right) / \delta_{j}$,

$$
\begin{aligned}
p+U_{1,0}(y)+\frac{1}{p} \omega_{1}(y)+\frac{1}{p^{2}} \omega_{2}(y) & \geq p+\log 8-2 \log \left(1+\frac{\varepsilon^{2} d\left(\xi_{j}\right)^{2}}{\delta_{j}^{2}}\right)+\frac{1}{4}+O\left(\frac{1}{p}\right) \\
& =p+\log 8-4 \log \frac{\varepsilon d\left(\xi_{j}\right)}{\delta_{j}}+\frac{1}{4}+O\left(\frac{\delta_{j}^{2}}{\varepsilon^{2} d\left(\xi_{j}\right)^{2}}\right)+O\left(\frac{1}{p}\right) \\
& =4 \log \frac{1}{\varepsilon}+\log \left(8 \mu_{j}^{4}\right)-4 \log \left(\lambda d\left(\xi_{j}\right)\right)+\frac{1}{4}+O\left(\frac{1}{p}\right) .
\end{aligned}
$$

By choosing $\varepsilon>0$ small enough, it is easy to check that $U_{\xi}>0$ in $B\left(\xi_{j}, \varepsilon d\left(\xi_{j}\right)\right)$, and $\sup _{B\left(\xi_{j}, \varepsilon d\left(\xi_{j}\right)\right)} U_{\xi} \rightarrow \sqrt{e}$ as $p$ and $\lambda$ go to $+\infty$ and satisfy (2.4). Moreover, by the maximum principle, we see that $G_{\lambda}\left(x, \xi_{j}\right)>0$ in $\bar{\Omega}$ and thus, by (2.16), $U_{\xi}$ is a positive uniformly bounded function in $\bar{\Omega}$. In conclusion, $0<U_{\xi} \leq 2 \sqrt{e}$ in $\bar{\Omega}$.

Let us define

$$
S_{p}(u)=\Delta u+u_{+}^{p}, \quad \text { where } u_{+}=\max \{u, 0\},
$$

and introduce the following functional:

$$
J_{p}^{\lambda}(u)=\frac{1}{2} \int_{\Omega}|\nabla u|^{2}-\frac{1}{p+1} \int_{\Omega} u_{+}^{p+1}+\frac{\lambda}{2} \int_{\partial \Omega} b(x) u^{2}, \quad u \in H^{1}(\Omega),
$$

whose nontrivial critical points are solutions of problem (1.1). Obviously, by the maximum principle, problem (1.1) is equivalent to

$$
S_{p}(u)=0, \quad u_{+} \not \equiv 0 \quad \text { in } \Omega, \quad \frac{\partial u}{\partial \nu}+\lambda b(x) u=0 \quad \text { on } \partial \Omega .
$$

We will seek solutions of problem (1.1) in the form $u=U_{\xi}+\phi$, where $\phi$ will represent a higher order correction. Observe that

$$
S_{p}\left(U_{\xi}+\phi\right)=L(\phi)+R_{\xi}+N(\phi)=0
$$

where

$$
\begin{gathered}
L(\phi)=\Delta \phi+W_{\xi} \phi \quad \text { with } \quad W_{\xi}=p U_{\xi}^{p-1}, \\
R_{\xi}=\Delta U_{\xi}+U_{\xi}^{p} \text { and } N(\phi)=\left(U_{\xi}+\phi\right)_{+}^{p}-U_{\xi}^{p}-p U_{\xi}^{p-1} \phi .
\end{gathered}
$$

In terms of $\phi$, problem (1.1) becomes

$$
\begin{cases}L(\phi)=-\left[R_{\xi}+N(\phi)\right] & \text { in } \Omega, \\ \frac{\partial \phi}{\partial v}+\lambda b(x) \phi=0 & \text { on } \partial \Omega .\end{cases}
$$

The main step in solving problem (2.22) is a solvability theory for the linear operator $L$ under a suitable choice of the points $\xi_{j}$. In developing this theory, we will take into account the invariance, under translations and dilations, of the problem $\Delta e^{v}+e^{v}=0$ in $\mathbb{R}^{2}$. We will apply the solvability theory for the linear operator $L$ in a weighted $L^{\infty}$-norm space, following $[9,13]$. For any $\xi=\left(\xi_{1}, \ldots, \xi_{m}\right) \in \mathcal{O}_{\varepsilon}$ and $h \in L^{\infty}(\Omega)$, define

$$
\|h\|_{*}=\sup _{x \in \Omega}\left|\left(\sum_{j=1}^{m} \frac{\delta_{j}}{\left(\delta_{j}^{2}+\left|x-\xi_{j}\right|^{2}\right)^{3 / 2}}\right)^{-1} h(x)\right| .
$$

We conclude this section by showing an estimate of $R_{\xi}$ in $\|\cdot\|_{\star}$. 
Proposition 2.3. Let $\varepsilon>0$ be fixed and small. There exist $C>0, p_{0}>0$ and $\lambda_{0}>0$ such that for any points $\xi=\left(\xi_{1}, \ldots, \xi_{m}\right) \in \mathcal{O}_{\varepsilon}$, and any $p \geq p_{0}$ and $\lambda \geq \lambda_{0}$ satisfying (2.4),

$$
\left\|R_{\xi}\right\| * \leq \frac{C}{p^{4}} .
$$

Proof. Observe that by (2.6)-(2.7) and (2.12)-(2.13),

$$
\begin{aligned}
\Delta U_{\xi} & =\sum_{j=1}^{m} \frac{1}{y \mu_{j}^{2 /(p-1)}}\left[-e^{U_{\delta_{j}, \xi_{j}}}+\frac{1}{p \delta_{j}^{2}} \Delta \omega_{1}\left(\frac{x-\xi_{j}}{\delta_{j}}\right)+\frac{1}{p^{2} \delta_{j}^{2}} \Delta \omega_{2}\left(\frac{x-\xi_{j}}{\delta_{j}}\right)\right] \\
& =\sum_{j=1}^{m} \frac{e^{U_{\delta_{j}, \xi_{j}}}}{y \mu_{j}^{2 /(p-1)}}\left[-1+\frac{1}{p} f_{1}\left(\frac{x-\xi_{j}}{\delta_{j}}\right)+\frac{1}{p^{2}} f_{2}\left(\frac{x-\xi_{j}}{\delta_{j}}\right)-\frac{1}{p} \omega_{1}\left(\frac{x-\xi_{j}}{\delta_{j}}\right)-\frac{1}{p^{2}} \omega_{2}\left(\frac{x-\xi_{j}}{\delta_{j}}\right)\right] .
\end{aligned}
$$

Far away from the points $\xi_{j}$, namely $\left|x-\xi_{j}\right| \geq \varepsilon d\left(\xi_{j}\right)$ for any $j=1, \ldots, m$, by (2.3)-(2.5), (2.16) and (2.25), we get

$$
\left(\sum_{k=1}^{m} \frac{\delta_{k}}{\left(\delta_{k}^{2}+\left|x-\xi_{k}\right|^{2}\right)^{3 / 2}}\right)^{-1}\left|\left(\Delta U_{\xi}+U_{\xi}^{p}\right)(x)\right| \leq \sum_{j=1}^{m} \frac{C}{\delta_{j}}\left(\frac{\delta_{j}^{2} \log ^{4} \delta_{j}}{y p^{2} d\left(\xi_{j}\right)^{4}}+\frac{1}{y^{p}}\right)=O\left(p \lambda^{3} e^{-p / 4}\right) .
$$

Let us now fix the index $j$ in $\{1, \ldots, m\}$ and the region $\left|x-\xi_{j}\right| \leq \varepsilon d\left(\xi_{j}\right)$. Taking into account (2.20) and the relation

$$
\left(\frac{p}{y \mu_{j}^{2 /(p-1)}}\right)^{p}=\frac{1}{y \delta_{j}^{2} \mu_{j}^{2 /(p-1)}}
$$

for $\left|x-\xi_{j}\right|=\delta_{j}|y| \leq \varepsilon d\left(\xi_{j}\right)$, we get

$$
U_{\xi}^{p}(x)=\frac{1}{y \delta_{j}^{2} \mu_{j}^{2 /(p-1)}}\left[1+\frac{1}{p} U_{1,0}(y)+\frac{1}{p^{2}} \omega_{1}(y)+\frac{1}{p^{3}}\left[\omega_{2}(y)+O\left(p^{2} \lambda \rho|y|\right)+O(p \lambda \rho)\right]\right]^{p} .
$$

From the Taylor expansions of the exponential and logarithmic function, we have

$$
\left(1+\frac{a}{p}+\frac{b}{p^{2}}+\frac{c}{p^{3}}\right)^{p}=e^{a}\left[1+\frac{1}{p}\left(b-\frac{a^{2}}{2}\right)+\frac{1}{p^{2}}\left(c-a b+\frac{a^{3}}{3}+\frac{b^{2}}{2}-\frac{a^{2} b}{2}+\frac{a^{4}}{8}\right)+O\left(\frac{\log ^{6}(|y|+2)}{p^{3}}\right)\right],
$$

which holds for $|y| \leq C e^{p / 8}$, provided that $-4 \log (|y|+2) \leq a(y) \leq C$ and $|b(y)|+|c(y)| \leq C \log (|y|+2)$. For $\left|x-\xi_{j}\right|=\delta_{j}|y| \leq \varepsilon d\left(\xi_{j}\right) \sqrt{\delta_{j}}$, we deduce that

$$
\begin{aligned}
U_{\xi}^{p}(x)= & \frac{1}{y \delta_{j}^{2} \mu_{j}^{2 /(p-1)}} e^{U_{1,0}(y)}\left[1+\frac{1}{p}\left(\omega_{1}-\frac{1}{2} U_{1,0}^{2}\right)+\frac{1}{p^{2}}\left(\omega_{2}-\omega_{1} U_{1,0}+\frac{1}{3} U_{1,0}^{3}+\frac{1}{2} \omega_{1}^{2}-\frac{1}{2} \omega_{1} U_{1,0}^{2}+\frac{1}{8} U_{1,0}^{4}\right)\right. \\
& \left.+O\left(\frac{\log ^{6}(|y|+2)}{p^{3}}\right)+O(\lambda \rho|y|)+O\left(\frac{\lambda \rho}{p}\right)\right] .
\end{aligned}
$$

Combining (2.1), (2.8), (2.25) and (2.30), for $\left|x-\xi_{j}\right|=\delta_{j}|y| \leq \varepsilon d\left(\xi_{j}\right) \sqrt{\delta_{j}}$, we have that

$$
\left(\Delta U_{\xi}+U_{\xi}^{p}\right)(x)=\frac{1}{y \delta_{j}^{2} \mu_{j}^{2 /(p-1)}} \frac{8}{\left(1+|y|^{2}\right)^{2}} O\left(\frac{\log ^{6}(|y|+2)}{p^{3}}\right),
$$

and therefore

$$
\begin{aligned}
\left(\sum_{k=1}^{m} \frac{\delta_{k}}{\left(\delta_{k}^{2}+\left|x-\xi_{k}\right|^{2}\right)^{3 / 2}}\right)^{-1}\left|\left(\Delta U_{\xi}+U_{\xi}^{p}\right)(x)\right| & \leq \frac{\left(\delta_{j}^{2}+\left|x-\xi_{j}\right|^{2}\right)^{3 / 2}}{\delta_{j}}\left|\left(\Delta U_{\xi}+U_{\xi}^{p}\right)(x)\right| \\
& \leq \frac{C}{y} \frac{1}{\left(1+|y|^{2}\right)^{1 / 2}} O\left(\frac{\log ^{6}(|y|+2)}{p^{3}}\right) \\
& \leq \frac{C}{p^{4}} .
\end{aligned}
$$


On the other hand, if $\varepsilon d\left(\xi_{j}\right) \sqrt{\delta_{j}} \leq\left|x-\xi_{j}\right|=\delta_{j}|y| \leq \varepsilon d\left(\xi_{j}\right)$, then by (2.28) we have

$$
U_{\xi}^{p}(x)=O\left(\frac{1}{y \delta_{j}^{2}} \frac{1}{\left(1+|y|^{2}\right)^{2}}\right),
$$

since $\left(1+\frac{s}{p}\right)^{p} \leq e^{s}$. Thus, in this region,

$$
\left(\sum_{k=1}^{m} \frac{\delta_{k}}{\left(\delta_{k}^{2}+\left|x-\xi_{k}\right|^{2}\right)^{3 / 2}}\right)^{-1}\left|\left(\Delta U_{\xi}+U_{\xi}^{p}\right)(x)\right| \leq \frac{1}{y} \frac{1}{\left(1+|y|^{2}\right)^{1 / 2}} O\left(\frac{1}{p^{2}} \log ^{4}|y|\right) \leq p \sqrt{\lambda} e^{-p / 8} .
$$

Joining together (2.26), (2.31) and (2.32), we obtain estimate (2.24).

\section{Analysis of the linearized operator}

In this section we prove the bounded invertibility of the operator $L$, uniformly on $\xi \in \mathcal{O}_{\varepsilon}$, by using the weighted $L^{\infty}$-norms introduced in (2.23). Let us recall that $L(\phi)=\Delta \phi+W_{\xi} \phi$, where $W_{\xi}(x)=p U_{\xi}^{p-1}(x)$. As in Proposition 2.3, for the potential $W_{\xi}(x)$ we have the following expansions.

Lemma 3.1. Let $\varepsilon>0$ be fixed and small. There exist $D_{0}>0, p_{0}>0$ and $\lambda_{0}>0$ such that

$$
W_{\xi}(x) \leq D_{0} \sum_{j=1}^{m} e^{U_{\delta_{j}, \xi_{j}}(x)}
$$

for any points $\xi=\left(\xi_{1}, \ldots, \xi_{m}\right) \in \mathcal{O}_{\varepsilon}$, and any $p \geq p_{0}$ and $\lambda \geq \lambda_{0}$ satisfying (2.4). Furthermore,

$$
W_{\xi}(x)=\frac{8}{\delta_{j}^{2}\left(1+|y|^{2}\right)^{2}}\left[1+\frac{1}{p}\left(\omega_{1}-U_{1,0}-\frac{1}{2} U_{1,0}^{2}\right)(y)+O\left(\frac{\log ^{4}(|y|+2)}{p^{2}}\right)\right]
$$

for any $\left|x-\xi_{j}\right| \leq \varepsilon d\left(\xi_{j}\right) \sqrt{\delta_{j}}$, where $y=\frac{x-\xi_{j}}{\delta_{j}}$.

Proof. If $\left|x-\xi_{j}\right|=\delta_{j}|y| \leq \varepsilon d\left(\xi_{j}\right)$ for some $j=1, \ldots, m$, then by (2.20) and (2.27),

$$
\begin{aligned}
W_{\xi}(x) & =p\left(\frac{p}{y \mu_{j}^{2 /(p-1)}}\right)^{p-1}\left[1+\frac{1}{p} U_{1,0}(y)+\frac{1}{p^{2}} \omega_{1}(y)+\frac{1}{p^{3}} \omega_{2}(y)+\frac{1}{p} O(\lambda \rho|y|)+\frac{1}{p^{2}} O(\lambda \rho)\right]^{p-1} \\
& =\frac{1}{\delta_{j}^{2}}\left[1+\frac{1}{p} U_{1,0}(y)+\frac{1}{p^{2}} \omega_{1}(y)+\frac{1}{p^{3}} \omega_{2}(y)+\frac{1}{p} O(\lambda \rho|y|)+\frac{1}{p^{2}} O(\lambda \rho)\right]^{p-1},
\end{aligned}
$$

where again we use the notation $y=\delta_{j}^{-1}\left(x-\xi_{j}\right)$. In this region, we have that

$$
W_{\xi}(x) \leq \frac{C}{\delta_{j}^{2}} e^{U_{1,0}(y)+O(\lambda \rho|y|)} e^{-\frac{1}{p}\left[U_{1,0}(y)+O(\lambda \rho|y|)\right]}=O\left(e^{U_{\delta_{j}, \xi_{j}}(x)}\right),
$$

since $\left(1+\frac{a}{p}\right)^{p-1} \leq e^{(p-1) a / p}$ and $U_{1,0}(y) \geq-p+O(1)$. In particular, from a slight modification of formula (2.29), that is,

$$
\left(1+\frac{a}{p}+\frac{b}{p^{2}}+\frac{c}{p^{3}}\right)^{p-1}=e^{a}\left[1+\frac{1}{p}\left(b-a-\frac{a^{2}}{2}\right)+O\left(\frac{\log ^{4}(|y|+2)}{p^{2}}\right)\right],
$$

we obtain that if $\left|x-\xi_{j}\right|=\delta_{j}|y| \leq \varepsilon d\left(\xi_{j}\right) \sqrt{\delta_{j}}$, then

$$
W_{\xi}(x)=\frac{8}{\delta_{j}^{2}\left(1+|y|^{2}\right)^{2}}\left[1+\frac{1}{p}\left(\omega_{1}-U_{1,0}-\frac{1}{2} U_{1,0}^{2}\right)(y)+O\left(\frac{\log ^{4}(|y|+2)}{p^{2}}\right)\right] .
$$

In addition, if $\left|x-\xi_{j}\right| \geq \varepsilon d\left(\xi_{j}\right)$ for all $j$, then by (2.16) we deduce

$$
W_{\xi}(x)=O\left(p\left(\frac{C}{y}\right)^{p-1}\right),
$$

which completes the proof. 
Remark 3.2. As for $W_{\xi}$, let us point out that if $\left|x-\xi_{j}\right| \leq \varepsilon d\left(\xi_{j}\right)$ for some $j=1, \ldots, m$, then

$$
p\left(U_{\xi}(x)+O\left(\frac{1}{p^{3}}\right)\right)^{p-2} \leq C p\left(\frac{p}{y \mu_{j}^{2 /(p-2)}}\right)^{p-2} e^{\frac{p-2}{p} U_{1,0}\left(\frac{x-\xi_{j}}{\delta_{j}}\right)}=O\left(e^{U_{\delta_{j}, \xi_{j}}(x)}\right) .
$$

Since this estimate is true if $\left|x-\xi_{j}\right| \geq \varepsilon d\left(\xi_{j}\right)$ for any $j=1, \ldots, m$, we have

$$
p\left(U_{\xi}(x)+O\left(\frac{1}{p^{3}}\right)\right)^{p-2} \leq C \sum_{j=1}^{m} e^{U_{\delta_{j}, \xi_{j}}(x)} .
$$

Let

$$
z_{0}(y)=\frac{|y|^{2}-1}{|y|^{2}+1}, \quad z_{i}(y)=4 \frac{y_{i}}{|y|^{2}+1}, \quad i=1,2 .
$$

It is well known that any bounded solution to

$$
\Delta \phi+\frac{8}{\left(1+|y|^{2}\right)^{2}} \phi=0 \quad \text { in } \mathbb{R}^{2}
$$

is a linear combination of $z_{i}, i=0,1,2$ (see [2]). Now we consider the following linear problem: given $h \in C(\bar{\Omega})$ and points $\xi=\left(\xi_{1}, \ldots, \xi_{m}\right) \in \mathcal{O}_{\varepsilon}$, we find a function $\phi \in H^{2}(\Omega)$ such that

$$
\begin{cases}L(\phi)=\Delta \phi+W_{\xi} \phi=h+\sum_{i=1}^{2} \sum_{j=1}^{m} c_{i j} e^{U_{\delta_{j}, \xi_{j}} Z_{i j}} & \text { in } \Omega, \\ \frac{\partial \phi}{\partial v}+\lambda b(x) \phi=0 & \text { on } \partial \Omega, \\ \int_{\Omega} e^{U_{\delta_{j}, \xi_{j}} Z_{i j} \phi=0} & \text { for } i=1,2, j=1, \ldots, m,\end{cases}
$$

for some coefficients $c_{i j}, i=1,2$ and $j=1, \ldots, m$. Here and in the sequel, for any $i=0,1,2$ and $j=1, \ldots, m$, we set

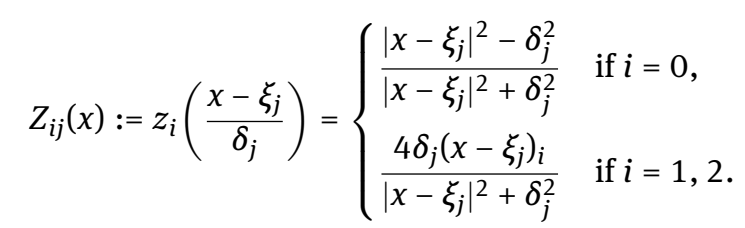

The main result of this section is the following proposition.

Proposition 3.3. Let $\varepsilon>0$ be fixed and small. There exist $p_{0}>0, \lambda_{0}>0$ and $C>0$ such that for any $h \in C(\bar{\Omega})$, any points $\xi=\left(\xi_{1}, \ldots, \xi_{m}\right) \in \mathcal{O}_{\varepsilon}$, and any $p>p_{0}$ and $\lambda>\lambda_{0}$ satisfying (2.4), there exists a unique solution $\phi$ to problem (3.2) for some scalars $c_{i j}, i=1,2, j=1, \ldots, m$, which satisfies

$$
\|\phi\|_{\infty} \leq C p\|h\|_{\star} .
$$

Proof. The proof of this result will be divided into six steps.

Step 1:

The operator $L$ satisfies the maximum principle in $\widetilde{\Omega}:=\Omega \backslash \bigcup_{j=1}^{m} B\left(\xi_{j}, R \delta_{j}\right)$ for $R$ large, independent of $p$ and $\lambda$. Specifically, if $\psi$ satisfies

$$
L(\psi)=\Delta \psi+W_{\xi} \psi \leq 0 \quad \text { in } \widetilde{\Omega}, \quad \psi \geq 0 \quad \text { on } \bigcup_{j=1}^{m} \partial B\left(\xi_{j}, R \delta_{j}\right) \text { and } \frac{\partial \psi}{\partial v}+\lambda b(x) \psi \geq 0 \quad \text { on } \partial \Omega,
$$

then $\psi \geq 0$ in $\widetilde{\Omega}$. To prove this, it suffices to construct a positive function $Z$ on $\widetilde{\Omega}$ such that

$$
L(Z)=\Delta Z+W_{\xi} Z<\quad \text { in } \widetilde{\Omega}, \quad Z>0 \quad \text { on } \bigcup_{j=1}^{m} \partial B\left(\xi_{j}, R \delta_{j}\right) \text { and } \frac{\partial Z}{\partial \nu}+\lambda b(x) Z>0 \quad \text { on } \partial \Omega .
$$


Indeed, let

$$
Z(x)=\sum_{j=1}^{m} z_{0}\left(\frac{a\left(x-\xi_{j}\right)}{\delta_{j}}\right), \quad a>0 .
$$

First, observe that if $\left|x-\xi_{j}\right| \geq R \delta_{j}$ for $R>\frac{1}{a}$, then $Z(x)>0$. On the other hand, since $Z(x) \leq m$,

$$
W_{\xi}(x) Z(x) \leq D_{0}\left(\sum_{j=1}^{m} e^{U_{\delta_{j}, \xi_{j}}(x)}\right) Z(x) \leq D_{0} Z(x) \sum_{j=1}^{m} \frac{8 \delta_{j}^{2}}{\left|x-\xi_{j}\right|^{4}} \leq m D_{0} \sum_{j=1}^{m} \frac{8 \delta_{j}^{2}}{\left|x-\xi_{j}\right|^{4}},
$$

where $D_{0}$ is the constant in Lemma 3.1. Further, by the definition of $z_{0}$,

$$
-\Delta Z(x)=\sum_{j=1}^{m} a^{2} \frac{8 \delta_{j}^{2}\left(a^{2}\left|x-\xi_{j}\right|^{2}-\delta_{j}^{2}\right)}{\left(a^{2}\left|x-\xi_{j}\right|^{2}+\delta_{j}^{2}\right)^{3}} \geq \frac{1}{3} \sum_{j=1}^{m} \frac{8 a^{2} \delta_{j}^{2}}{\left(a^{2}\left|x-\xi_{j}\right|^{2}+\delta_{j}^{2}\right)^{2}} \geq \frac{4}{27} \sum_{j=1}^{m} \frac{8 \delta_{j}^{2}}{a^{2}\left|x-\xi_{j}\right|^{4}},
$$

provided that $R>\frac{\sqrt{3}}{a}$. Thus, if $a$ is taken small and fixed but independent of $p$ and $\lambda$, and $R$ is chosen sufficiently large depending on this $a$, then we have that

$$
L(Z)=\Delta Z+W_{\xi} Z \leq\left(-\frac{4}{27} \frac{1}{a^{2}}+m D_{0}\right) \sum_{j=1}^{m} \frac{8 \delta_{j}^{2}}{\left|x-\xi_{j}\right|^{4}}<0 .
$$

Moreover,

$$
\left|\frac{\partial}{\partial v} Z(x)\right| \leq \sum_{j=1}^{m} \frac{C \delta_{j}^{2}}{a^{2}\left|x-\xi_{j}\right|^{3}}=O\left(\frac{\rho^{2}}{a^{2}} \sum_{j=1}^{m} \frac{1}{d\left(\xi_{j}\right)^{3}}\right) \quad \text { on } \partial \Omega
$$

and

$$
Z(x) \geq \frac{1}{2} \quad \text { on } \partial \Omega \cup\left(\bigcup_{j=1}^{m} \partial B\left(\xi_{j}, R \delta_{j}\right)\right),
$$

which, together with (2.3)-(2.5), deduce that on $\partial \Omega$,

$$
\frac{\partial Z}{\partial \nu}+\lambda b(x) Z \geq O\left(\frac{1}{a^{2}} \lambda^{3} \rho^{2}\right)+\frac{1}{2} \lambda b(x) \geq O\left(\lambda e^{-p / 2}\right)+\frac{1}{2} \lambda \min _{x \in \partial \Omega} b(x)>0,
$$

provided that $p$ and $\lambda$ are chosen sufficiently large. The function $Z(x)$ is what we are looking for.

Step 2:

Let $R$ and $\varepsilon_{0}$ be as before. We define the "inner norm" of $\phi$ as

$$
\|\phi\|_{i}=\sup _{x \in \bigcup_{j=1}^{m} B\left(\zeta_{j}, R \delta_{j}\right)}|\phi|(x),
$$

and claim that there exists a constant $C>0$ such that if $L(\phi)=h$ in $\Omega$ and $\frac{\partial \phi}{\partial v}+\lambda b(x) \phi=g$ on $\partial \Omega$, then

$$
\|\phi\|_{L^{\infty}(\Omega)} \leq C\left(\|\phi\|_{i}+\|h\|_{\star}+\frac{1}{\lambda}\|g\|_{L^{\infty}(\partial \Omega)}\right)
$$

for any $h \in C^{0, \alpha}(\bar{\Omega})$ and $g \in C^{0, \alpha}(\partial \Omega)$. We will establish this estimate with the use of suitable barriers. Let $M=2$ diam $\Omega$. Consider the solution $\psi_{j}(x)$ of the problem

$$
\begin{cases}-\Delta \psi_{j}=\frac{2 \delta_{j}}{\left|x-\xi_{j}\right|^{3}} & \text { in } R \delta_{j}<\left|x-\xi_{j}\right|<M, \\ \psi_{j}(x)=0 & \text { on }\left|x-\xi_{j}\right|=R \delta_{j} \text { and }\left|x-\xi_{j}\right|=M .\end{cases}
$$

The function $\psi_{j}(x)$ is the positive function given by

$$
\psi_{j}=-\frac{2 \delta_{j}}{\left|x-\xi_{j}\right|}+A+B \log \left|x-\xi_{j}\right|,
$$


where

$$
B=2\left(\frac{\delta_{j}}{M}-\frac{1}{R}\right) \frac{1}{\log \left(\frac{M}{R \delta_{j}}\right)}<0 \text { and } A=\frac{2 \delta_{j}}{M}-B \log M .
$$

Observe that for $R \delta_{j} \leq\left|x-\xi_{j}\right| \leq M$,

$$
\psi_{j}(x) \leq A+B \log \left(R \delta_{j}\right)=\frac{2 \delta_{j}}{M}-B \log \frac{M}{R \delta_{j}}=\frac{2}{R} .
$$

Thus, $\psi_{j}(x)$ is uniformly bounded from above by a constant independent of $p$ and $\lambda$. In view of

$$
\left|\nabla \psi_{j}(x)\right|=O\left(\frac{\rho}{\left|x-\xi_{j}\right|^{2}}+\frac{1}{|\log \rho|} \frac{1}{\left|x-\xi_{j}\right|}\right),
$$

and by (2.3)-(2.5), we get

$$
\left|\frac{\partial}{\partial \nu} \psi_{j}(x)\right|=O\left(\lambda e^{-p / 4}+\frac{\lambda}{p}\right) \quad \text { on } \partial \Omega
$$

Now let

$$
\widetilde{\phi}(x)=C_{0}\left(2 Z(x)+\sum_{j=1}^{m} \psi_{j}(x)\right)\left(\|\phi\|_{i}+\|h\|_{\star}+\frac{1}{\lambda}\|g\|_{L^{\infty}(\partial \Omega)}\right),
$$

where $Z$ was defined in the previous step, and $C_{0}>2$ is chosen larger if necessary. First of all, observe that for $x \in \bigcup_{j=1}^{m} \partial B\left(\xi_{j}, R \delta_{j}\right)$, by the definition of $Z$,

$$
\widetilde{\phi}(x) \geq 2 C_{0}\|\phi\|_{i} Z(x) \geq\|\phi\|_{i} \geq|\phi|(x) .
$$

For $x \in \partial \Omega$, by (3.5), (3.7) and the positivity of $Z(x)$ and $\psi_{j}(x)$,

$$
\begin{aligned}
\frac{\partial \widetilde{\phi}}{\partial v}(x)+\lambda b(x) \widetilde{\phi}(x) & \geq\left[O\left(\lambda e^{-p / 2}+\lambda e^{-p / 4}+\frac{\lambda}{p}\right)+C_{0} \lambda \min _{x \in \partial \Omega} b(x)\right]\left(\|\phi\|_{i}+\|h\|_{\star}+\frac{1}{\lambda}\|g\|_{L^{\infty}(\partial \Omega)}\right) \\
& \geq \frac{1}{2} C_{0} \lambda\left(\min _{x \in \partial \Omega} b(x)\right)\left(\|\phi\|_{i}+\|h\|_{\star}+\frac{1}{\lambda}\|g\|_{L^{\infty}(\partial \Omega)}\right) \\
& \geq|g(x)| .
\end{aligned}
$$

Finally, for $x \in \Omega \backslash \bigcup_{j=1}^{m} B\left(\xi_{j}, R \delta_{j}\right)$, by (3.1), (3.6) and the definition of $\|\cdot\| \star$ in (2.23),

$$
\begin{aligned}
L(\widetilde{\phi}) & \leq C_{0}\left(\|\phi\|_{i}+\|h\|_{\star}+\frac{1}{\lambda}\|g\|_{L^{\infty}(\partial \Omega)}\right) \sum_{j=1}^{m} L\left(\psi_{j}\right)(x) \\
& =C_{0}\left(\|\phi\|_{i}+\|h\|_{\star}+\frac{1}{\lambda}\|g\|_{L^{\infty}(\partial \Omega)}\right) \sum_{j=1}^{m}\left(-\frac{2 \delta_{j}}{\left|x-\xi_{j}\right|^{3}}+W_{\xi}(x) \psi_{j}(x)\right) \\
& \leq C_{0}\left(\|\phi\|_{i}+\|h\|_{\star}+\frac{1}{\lambda}\|g\|_{L^{\infty}(\partial \Omega)}\right) \sum_{j=1}^{m}\left(-\frac{2 \delta_{j}}{\left|x-\xi_{j}\right|^{3}}+\frac{2 m D_{0}}{R} e^{U_{\delta_{j}, \xi_{j}}(x)}\right) \\
& \leq-C_{0}\|h\|_{\star} \sum_{j=1}^{m} \frac{\delta_{j}}{\left(\delta_{j}^{2}+\left|x-\xi_{j}\right|^{2}\right)^{3 / 2}} \\
& \leq-|h(x)| \\
& \leq-|L(\phi)|(x),
\end{aligned}
$$

provided that $R>16 m D_{0}$, and $p$ and $\lambda$ are large enough. Hence, by the maximum principle in Step 1, we obtain

$$
|\phi(x)| \leq \widetilde{\phi}(x) \text { for } x \in \widetilde{\Omega},
$$

and therefore, since $Z(x) \leq m$ and $\psi_{j}(x) \leq \frac{2}{R}$,

$$
\|\phi\|_{L^{\infty}(\Omega)} \leq C\left(\|\phi\|_{i}+\|h\|_{\star}+\frac{1}{\lambda}\|g\|_{L^{\infty}(\partial \Omega)}\right) .
$$


Step 3:

We prove uniform a priori estimates for the solutions $\phi$ of the problem

$$
\begin{cases}L(\phi)=h & \text { in } \Omega, \\ \frac{\partial \phi}{\partial \nu}+\lambda b(x) \phi=g & \text { on } \partial \Omega,\end{cases}
$$

where $h \in C^{0, \alpha}(\bar{\Omega})$ and $g \in C^{0, \alpha}(\partial \Omega)$, and in addition $\phi$ satisfy the orthogonality conditions

$$
\int_{\Omega} e^{U_{\delta_{j}, r_{j}}} Z_{i j} \phi=0 \text { for } i=0,1,2, j=1, \ldots, m \text {. }
$$

Namely, we prove that there exists a positive constant $C$ such that for any points $\xi=\left(\xi_{1}, \ldots, \xi_{m}\right) \in \mathcal{O}_{\varepsilon}$, $h \in C^{0, \alpha}(\bar{\Omega})$ and $g \in C^{0, \alpha}(\partial \Omega)$,

$$
\|\phi\|_{L^{\infty}(\Omega)} \leq C\left(\|h\|_{\star}+\frac{1}{\lambda}\|g\|_{L^{\infty}(\partial \Omega)}\right)
$$

for $p$ and $\lambda$ sufficiently large. By contradiction, assume the existence of sequences $p_{n} \rightarrow+\infty, \lambda_{n} \rightarrow+\infty$, of points $\xi^{n}=\left(\xi_{1}^{n}, \ldots, \xi_{m}^{n}\right) \in \mathcal{O}_{\varepsilon}$, and of functions $h_{n}, g_{n}$ and associated solutions $\phi_{n}$ such that $\left\|h_{n}\right\|_{*} \rightarrow 0$, $\frac{1}{\lambda_{n}}\left\|g_{n}\right\|_{L^{\infty}(\partial \Omega)} \rightarrow 0$ and $\left\|\phi_{n}\right\|_{L^{\infty}(\Omega)}=1$.

Since $\left\|\phi_{n}\right\|_{L^{\infty}(\Omega)}=1$, Step 2 shows that $\liminf _{n \rightarrow+\infty}\left\|\phi_{n}\right\|_{i}>0$. Set $\widehat{\phi}_{j}^{n}(y)=\phi_{n}\left(\delta_{j}^{n} y+\xi_{j}^{n}\right)$ for $j=1, \ldots, m$. Observe that by (2.3), (2.5) and (2.18),

$$
\min _{j=1, \ldots, m} \frac{\operatorname{dist}\left(\xi_{j}^{n}, \partial \Omega\right)}{\delta_{j}^{n}} \rightarrow+\infty,
$$

which means that the domain of definition of $\widehat{\phi}_{j}^{n}$ approaches $\mathbb{R}^{2}$ as $n \rightarrow+\infty$. By Lemma 3.1, elliptic estimates readily imply that $\widehat{\phi}_{j}^{n}$ converges uniformly over compact sets to a bounded solution $\widehat{\phi}_{j}^{\infty}$ of

$$
\Delta \phi+\frac{8}{\left(1+|y|^{2}\right)^{2}} \phi=0 \quad \text { in } \mathbb{R}^{2} .
$$

This implies that $\widehat{\phi}_{j}^{\infty}$ is a linear combination of the functions $z_{i}, i=0,1,2$. Since $\left\|\widehat{\phi}_{j}^{n}\right\|_{L^{\infty}(\Omega)} \leq 1$, by Lebesgue's theorem, the orthogonality conditions on $\widehat{\phi}_{j}^{n}$ pass to the limit and give

$$
\int_{\mathbb{R}^{2}} \frac{8}{\left(1+|y|^{2}\right)^{2}} z_{i}(y) \widehat{\phi}_{j}^{\infty} d y=0 \text { for } i=0,1,2 .
$$

Hence, $\widehat{\phi}_{j}^{\infty} \equiv 0$ for any $j=1, \ldots, m$ contradicting $\lim \inf _{n \rightarrow+\infty}\left\|\phi_{n}\right\|_{i}>0$.

Step 4:

We prove that for any solution $\phi$ of the problem

$$
\begin{cases}L(\phi)=h & \text { in } \Omega, \\ \frac{\partial \phi}{\partial \nu}+\lambda b(x) \phi=0 & \text { on } \partial \Omega,\end{cases}
$$

which in addition satisfies the orthogonality conditions

$$
\int_{\Omega} e^{U_{\delta_{j}, \xi_{j}}} Z_{i j} \phi=0 \text { for } i=1,2, j=1, \ldots, m,
$$

there exists a positive constant $C>0$ such that

$$
\|\phi\|_{L^{\infty}(\Omega)} \leq C p\|h\|_{\star}
$$


for $h \in C^{0, \alpha}(\bar{\Omega})$. Proceeding by contradiction, as in Step 3, we can suppose further that

$$
\left\|\phi_{n}\right\|_{L^{\infty}(\Omega)}=1 \text { and } p_{n}\left\|h_{n}\right\|_{*} \rightarrow 0 \quad \text { as } n \rightarrow+\infty
$$

but we lose the condition

$$
\int_{\mathbb{R}^{2}} \frac{8}{\left(1+|y|^{2}\right)^{2}} z_{0}(y) \widehat{\phi}_{j}^{\infty}=0
$$

in the limit. Hence, we have that

$$
\widehat{\phi}_{j}^{n} \rightarrow \widehat{\phi}_{j}^{\infty}=C_{j} \frac{|y|^{2}-1}{|y|^{2}+1} \text { in } C_{\text {loc }}^{0}\left(\mathbb{R}_{+}^{2}\right)
$$

for some constants $C_{j}$. To reach a contradiction, we have to show that $C_{j}=0$ for any $j=1, \ldots, m$. We will obtain it from the stronger condition (3.8) on $h_{n}$.

To this end, we perform the following construction. According to [4, 13], there exist radial solutions $\omega$ and $\zeta$, respectively, of the equations

$$
\Delta \omega+\frac{8}{\left(1+|y|^{2}\right)^{2}} \omega=\frac{8}{\left(1+|y|^{2}\right)^{2}} z_{0}(y) \text { and } \Delta \zeta+\frac{8}{\left(1+|y|^{2}\right)^{2}} \zeta=\frac{8}{\left(1+|y|^{2}\right)^{2}} \quad \text { in } \mathbb{R}^{2},
$$

such that

$$
\omega(y)=\frac{4}{3} \log |y|+O\left(\frac{1}{|y|}\right) \text { and } \zeta(y)=O\left(\frac{1}{|y|}\right) \quad \text { as }|y| \rightarrow+\infty
$$

and

$$
\nabla \omega(y)=\frac{4}{3} \cdot \frac{y}{1+|y|^{2}}+O\left(\frac{1}{1+|y|^{2}}\right) \text { and } \nabla \zeta(y)=O\left(\frac{1}{1+|y|^{2}}\right) \quad \text { for all } y \in \mathbb{R}^{2}
$$

since

$$
8 \int_{0}^{+\infty} r \frac{\left(r^{2}-1\right)^{2}}{\left(r^{2}+1\right)^{4}} d r=\frac{4}{3} \text { and } 8 \int_{0}^{+\infty} r \frac{r^{2}-1}{\left(r^{2}+1\right)^{3}} d r=0 .
$$

For simplicity, from now on we omit the dependence on $n$. For $j=1, \ldots, m$, define now

$$
u_{j}(x)=\omega\left(\frac{x-\xi_{j}}{\delta_{j}}\right)+\frac{4}{3}\left(\log \delta_{j}\right) Z_{0 j}(x)+\frac{8 \pi}{3} H_{\lambda}\left(\xi_{j}, \xi_{j}\right) \zeta\left(\frac{x-\xi_{j}}{\delta_{j}}\right),
$$

and denote its projection $P u_{j}=u_{j}+\widetilde{H}_{j}$, where $\widetilde{H}_{j}$ is a correction term defined as the solution of

$$
\begin{cases}-\Delta \widetilde{H}_{j}=0 & \text { in } \Omega, \\ \frac{\partial \widetilde{H}_{j}}{\partial \nu}+\lambda b(x) \widetilde{H}_{j}=-\frac{\partial u_{j}}{\partial v}-\lambda b(x) u_{j} & \text { on } \partial \Omega .\end{cases}
$$

Observe that on $\partial \Omega$,

$$
\left(\frac{\partial}{\partial v}+\lambda b(x)\right)\left(\widetilde{H}_{j}+\frac{8 \pi}{3} H_{\lambda}\left(x, \xi_{j}\right)\right)=O\left(\lambda^{2} \rho\right)+\left(\log \delta_{j}\right) O\left(\lambda^{3} \rho^{2}\right)+H_{\lambda}\left(\xi_{j}, \xi_{j}\right) O\left(\lambda^{2} \rho\right) .
$$

By (1.5)-(1.8) and the maximum principle with Robin boundary condition, we get

$$
\begin{cases}P u_{j}=u_{j}-\frac{8 \pi}{3} H_{\lambda}\left(x, \xi_{j}\right)+O(\lambda \rho \log \lambda) & \text { in } C(\bar{\Omega}), \\ P u_{j}=-\frac{8 \pi}{3} G_{\lambda}\left(x, \xi_{j}\right)+O\left(\lambda \rho \log \lambda+\frac{\rho \log \lambda}{\left|x-\xi_{j}\right|}+\frac{\rho^{2} \log \rho}{\left|x-\xi_{j}\right|^{2}}\right) & \text { in } C_{\mathrm{loc}}\left(\bar{\Omega} \backslash\left\{\xi_{j}\right\}\right) .\end{cases}
$$

The function $P u_{j}$ solves

$$
\begin{cases}\Delta P u_{j}+W_{\xi} P u_{j}=e^{U_{\delta_{j}, \xi_{j}}} Z_{0 j}+\left(W_{\xi}-e^{U_{\delta_{j}, \xi_{j}}}\right) P u_{j}+R_{j} & \text { in } \Omega, \\ \frac{\partial}{\partial \nu} P u_{j}+\lambda b(x) P u_{j}=0 & \text { on } \partial \Omega,\end{cases}
$$


where

$$
R_{j}(x)=\left(P u_{j}-u_{j}+\frac{8 \pi}{3} H_{\lambda}\left(\xi_{j}, \xi_{j}\right)\right) e^{U_{\delta_{j}, \xi_{j}}} .
$$

Multiply (3.11) by $\phi$ and integrate by parts to obtain

$$
\int_{\Omega} e^{U_{\delta_{j}, \xi_{j}}} Z_{0 j} \phi+\int_{\Omega}\left(W_{\xi}-e^{U_{\delta_{j}, \xi_{j}}}\right) P u_{j} \phi=\int_{\Omega} P u_{j} h-\int_{\Omega} R_{j} \phi .
$$

We estimate each term of (3.13). First of all, by Lebesgue’s theorem and (3.9) we get

$$
\int_{\Omega} e^{U_{\delta_{j}, \xi_{j}}} Z_{0 j} \phi \rightarrow C_{j} \int_{\mathbb{R}^{2}} \frac{8\left(|y|^{2}-1\right)^{2}}{\left(|y|^{2}+1\right)^{4}} d y=\frac{8 \pi}{3} C_{j} .
$$

Note that for fixed small $\delta>0$, there exists a constant $C>0$ such that for all $\left|x-\xi_{j}\right|=\delta$ and $\lambda d(x)=O(1)$,

$$
G_{\lambda}\left(x, \xi_{j}\right) \geq \frac{C}{\delta \lambda},
$$

provided that $\lambda$ is sufficiently large (see [8, p. 2685]). Thus, by Lemma 3.1, (2.4) and (3.10), we have

$$
\begin{aligned}
& \int_{\Omega}\left(W_{\xi}-e^{\left.U_{\delta_{j}, \xi_{j}}\right) P u_{j} \phi=} \int_{B\left(\xi_{j}, \varepsilon d\left(\xi_{j}\right) \sqrt{\delta_{j}}\right)}\left(W_{\xi}-e^{\left.U_{\delta_{j}, \xi_{j}}\right) P u_{j} \phi-\frac{8 \pi}{3} \sum_{k=j} G_{\lambda}\left(\xi_{k}, \xi_{j}\right)} \int_{B\left(\xi_{k}, \varepsilon d\left(\xi_{k}\right) \sqrt{\delta_{k}}\right)} W_{\xi} \phi+O\left(\lambda^{2} \sqrt{\rho}+\lambda \rho \log \lambda\right)\right.\right. \\
& =\int_{B\left(0, \varepsilon d\left(\xi_{j}\right) / \sqrt{\delta_{j}}\right)} \frac{8}{\left(1+|y|^{2}\right)^{2}} \frac{1}{p}\left(\omega_{1}-U_{1,0}-\frac{1}{2} U_{1,0}^{2}\right) \frac{4}{3}\left(\log \delta_{j}\right) z_{0}(y) \widehat{\phi}_{j} \\
& -\frac{8 \pi}{3} \sum_{k=j} G_{\lambda}\left(\xi_{k}, \xi_{j}\right) \int_{B\left(0, \varepsilon d\left(\xi_{k}\right) / \sqrt{\delta_{k}}\right)} \frac{8}{\left(1+|y|^{2}\right)^{2}} \widehat{\phi}_{k}+O\left(\frac{1}{p}\right) \\
& =-\frac{C_{j}}{3} \int_{\mathbb{R}^{2}} \frac{8\left(|y|^{2}-1\right)^{2}}{\left(1+|y|^{2}\right)^{4}}\left(\omega_{1}-U_{1,0}-\frac{1}{2} U_{1,0}^{2}\right)(y)+o(1),
\end{aligned}
$$

since Lebesgue's theorem and (3.9) imply

$$
\int_{B\left(0, \varepsilon d\left(\xi_{j}\right) / \sqrt{\delta_{j}}\right)} \frac{8}{\left(1+|y|^{2}\right)^{2}}\left(\omega_{1}-U_{1,0}-\frac{1}{2} U_{1,0}^{2}\right) z_{0}(y) \widehat{\phi}_{j} \rightarrow C_{j} \int_{\mathbb{R}^{2}} \frac{8\left(|y|^{2}-1\right)^{2}}{\left(1+|y|^{2}\right)^{4}}\left(\omega_{1}-U_{1,0}-\frac{1}{2} U_{1,0}^{2}\right)
$$

and

$$
\int_{B\left(0, \varepsilon d\left(\xi_{k}\right) / \sqrt{\delta_{k}}\right)} \frac{8}{\left(1+|y|^{2}\right)^{2}} \widehat{\phi}_{k} \rightarrow C_{k} \int_{\mathbb{R}^{2}} \frac{8}{\left(1+|y|^{2}\right)^{2}} \frac{|y|^{2}-1}{|y|^{2}+1}=0 .
$$

Using (2.10), in a straightforward but tedious way, we can compute

$$
\int_{\mathbb{R}^{2}} \frac{8\left(|y|^{2}-1\right)^{2}}{\left(1+|y|^{2}\right)^{4}}\left(\omega_{1}-U_{1,0}-\frac{1}{2} U_{1,0}^{2}\right)(y) d y=-8 \pi
$$

(see [13, p. 50]). So we obtain

$$
\int_{\Omega}\left(W_{\xi}-e^{U_{\delta_{j}, \xi_{j}}}\right) P u_{j} \phi=\frac{8 \pi}{3} C_{j}+o(1) .
$$


As for the right-hand side of (3.13), by (2.4), (2.23) and (3.10), we have that

$$
\begin{aligned}
\left|\int_{\Omega} P u_{j} h\right| & \leq C\|h\|_{\star} \int_{\Omega} \sum_{k=1}^{m} \frac{\delta_{k}}{\left(\delta_{k}^{2}+\left|x-\xi_{k}\right|^{2}\right)^{3 / 2}}\left|P u_{j}\right| d x \\
& =C\|h\|_{\star} \sum_{k=1}^{m} \int_{\Omega_{\delta_{k}}} \frac{1}{\left(1+|y|^{2}\right)^{3 / 2}}\left|P u_{j}\left(\delta_{k} y+\xi_{k}\right)\right| d y \\
& \leq C\|h\|_{\star} \int_{\mathbb{R}^{2}} \frac{\log (|y|+2)}{\left(1+|y|^{2}\right)^{3 / 2}} d y+C p\|h\|_{\star} \int_{\mathbb{R}^{2}} \frac{1}{\left(1+|y|^{2}\right)^{3 / 2}} d y \\
& \leq C p\|h\|_{\star},
\end{aligned}
$$

where $\Omega_{\delta_{k}}:=\frac{1}{\delta_{k}}\left(\Omega-\left\{\xi_{k}\right\}\right)$. Finally, using (2.15) and the maximum principle with Robin boundary condition, we find that $\left|\nabla H_{\lambda}\left(x, \xi_{j}\right)\right|=O(\lambda \log \lambda)$ holds uniformly in $\Omega$. By (3.10) and (3.12), we deduce

$$
\int_{\Omega} R_{j} \phi=O\left(\int_{\Omega} e^{U_{\delta_{j}, \xi_{j}}}\left(\left|x-\xi_{j}\right| \lambda \log \lambda+\lambda \rho \log \lambda\right) d x\right)=O\left(e^{-p / 4} \log \lambda\right) .
$$

Hence, inserting (3.14), (3.16), (3.17) and (3.18) in (3.13), and taking into account (3.8), we conclude that

$$
\frac{16 \pi}{3} C_{j}=o(1) \text { for any } j=1, \ldots, m \text {. }
$$

Necessarily, $C_{j}=0$, a contradiction and the claim is proved.

\section{Step 5:}

We establish the validity of the a priori estimate

$$
\|\phi\|_{\infty} \leq C p\|h\|_{\star}
$$

for solutions of problem (3.2) and $h \in C^{0, \alpha}(\bar{\Omega})$. Step 4 gives

$$
\|\phi\|_{L^{\infty}(\Omega)} \leq C p\left(\|h\|_{\star}+\sum_{i=1}^{2} \sum_{j=1}^{m}\left|c_{i j}\right| \cdot\left\|e^{U_{\delta_{j}, \xi_{j}}} Z_{i j}\right\|_{\star}\right) \leq C p\left(\|h\|_{\star}+\sum_{i=1}^{2} \sum_{j=1}^{m}\left|c_{i j}\right|\right) .
$$

As before, arguing by contradiction of (3.19), we can proceed, as in Step 3, and suppose further that

$$
\left\|\phi_{n}\right\|_{L^{\infty}(\Omega)}=1, \quad p_{n}\left\|h_{n}\right\|_{\star} \rightarrow 0 \text { and } p_{n} \sum_{i=1}^{2} \sum_{j=1}^{m}\left|c_{i j}^{n}\right| \geq \delta>0 \quad \text { as } n \rightarrow+\infty .
$$

We omit the dependence on $n$. It suffices to estimate the values of the constants $c_{i j}$. To this end, we define $P Z_{i j}$ as the projection of $Z_{i j}$ under homogeneous Robin boundary condition, namely

$$
\begin{cases}\Delta P Z_{i j}=\Delta Z_{i j}=-e^{U_{\delta_{j},}, \xi_{j}} Z_{i j} & \text { in } \Omega \\ \frac{\partial P Z_{i j}}{\partial v}+\lambda b(x) P Z_{i j}=0 & \text { on } \partial \Omega\end{cases}
$$

As in Lemma 2.1, for $i=1,2$ and $j=1, \ldots, m$, we have the following expansions:

$$
P Z_{i j}=Z_{i j}+8 \pi \delta_{j} \partial_{\left(\xi_{j}\right)} H_{\lambda}\left(\cdot, \xi_{j}\right)+O\left(\lambda^{3} \rho^{3}\right) \text { and } P Z_{0 j}=Z_{0 j}-1+O\left(\lambda^{2} \rho^{2}\right)
$$

in $C(\bar{\Omega})$ and in $C_{\text {loc }}^{2}(\Omega)$, and

$$
P Z_{i j}=8 \pi \delta_{j} \partial_{\left(\xi_{j}\right)_{i}} G_{\lambda}\left(\cdot, \xi_{j}\right)+O\left(\lambda^{3} \rho^{3}+\frac{\rho^{3}}{\left|x-\xi_{j}\right|^{3}}\right) \text { and } P Z_{0 j}=O\left(\lambda^{2} \rho^{2}+\frac{\rho^{2}}{\left|x-\xi_{j}\right|^{2}}\right)
$$


in $C\left(\bar{\Omega} \backslash\left\{\xi_{j}\right\}\right)$ and in $C_{\text {loc }}^{2}\left(\Omega \backslash\left\{\xi_{j}\right\}\right)$. By (3.21) and (3.22), we deduce the following "orthogonality" relations: for each $i, l=1,2$ and $j, k=1, \ldots, m$,

$$
\int_{\Omega} e^{U_{\delta_{j}, \xi_{j}}} Z_{i j} P Z_{l k}=\left(64 \int_{\mathbb{R}^{2}} \frac{|y|^{2}}{\left(1+|y|^{2}\right)^{4}}\right) \delta_{j k} \delta_{i l}+O(\lambda \rho),
$$

and

$$
\int_{\Omega} e^{U_{\delta_{j}, \xi_{j}}} Z_{0 j} P Z_{l k}=O(\lambda \rho)
$$

uniformly for any points $\xi=\left(\xi_{1}, \ldots, \xi_{m}\right) \in \mathcal{O}_{\varepsilon}$, where $\delta_{j k}$ and $\delta_{i l}$ denote Kronecker's symbols. Indeed, we have

$$
\begin{aligned}
\int_{\Omega} e^{U_{\delta_{j}, \xi_{j}}} Z_{i j} P Z_{l j} & =\int_{B\left(\xi_{j}, \varepsilon d\left(\xi_{j}\right)\right)} e^{U_{\delta_{j}, \xi_{j}}} Z_{i j}\left[Z_{l j}+8 \pi \delta_{j} \frac{\partial H_{\lambda}}{\partial\left(\xi_{j}\right)_{l}}\left(x, \xi_{j}\right)+O\left(\lambda^{3} \rho^{3}\right)\right] d x+O\left(\lambda^{4} \rho^{4}\right) \\
& =\int_{B\left(0, \varepsilon d\left(\xi_{j}\right) / \delta_{j}\right)} \frac{8}{\left(1+|y|^{2}\right)^{2}} \frac{4 y_{i}}{1+|y|^{2}}\left[\frac{4 y_{l}}{1+|y|^{2}}+O(\lambda \rho)\right] d y+O\left(\lambda^{4} \rho^{4}\right) \\
& =\left(64 \int_{\mathbb{R}^{2}} \frac{|y|^{2}}{\left(1+|y|^{2}\right)^{4}}\right) \delta_{i l}+O(\lambda \rho), \\
\int_{\Omega} e^{U_{\delta_{j}, \xi_{j}} Z_{0 j} P Z_{l j}} & =\int_{B\left(\xi_{j}, \varepsilon d\left(\xi_{j}\right)\right)} e^{U_{\delta_{j}, \xi_{j}} Z_{0 j}\left[Z_{l j}+8 \pi \delta_{j} \frac{\partial H_{\lambda}}{\partial\left(\xi_{j}\right)_{l}}\left(x, \xi_{j}\right)+O\left(\lambda^{3} \rho^{3}\right)\right] d x+O\left(\lambda^{3} \rho^{3}\right)} \\
& =\int_{B\left(0, \varepsilon d\left(\xi_{j}\right) / \delta_{j}\right)} \frac{8}{\left(1+|y|^{2}\right)^{2}} \frac{|y|^{2}-1}{|y|^{2}+1}\left[\frac{4 y_{l}}{1+|y|^{2}}+O(\lambda \rho)\right] d y+O\left(\lambda^{3} \rho^{3}\right) \\
& =O(\lambda \rho),
\end{aligned}
$$

and for $j=k$,

$$
\begin{aligned}
\int_{\Omega} e^{U_{\delta_{j}, \xi_{j}}} Z_{i j} P Z_{l k} & =\int_{B\left(\xi_{j}, \varepsilon d\left(\xi_{j}\right)\right)} e^{U_{\delta_{j}, \xi_{j}}} Z_{i j}\left[8 \pi \delta_{k} \frac{\partial G_{\lambda}}{\partial\left(\xi_{k}\right)_{l}}\left(x, \xi_{k}\right)+O\left(\lambda^{3} \rho^{3}+\frac{\rho^{3}}{\left|x-\xi_{k}\right|^{3}}\right)\right] d x+O\left(\lambda^{6} \rho^{4}\right) \\
& =O\left(\lambda \rho \int_{B\left(\xi_{j}, \varepsilon d\left(\xi_{j}\right)\right)} e^{\left.U_{\delta_{j}, \xi_{j}} Z_{i j} d x\right)+O\left(\lambda^{6} \rho^{4}\right)}\right. \\
& =O(\lambda \rho), \\
\int_{\Omega} e^{U_{\delta_{j}, \xi_{j}} Z_{0 j} P Z_{l k}} & =\int_{B\left(\xi_{j}, \varepsilon d\left(\xi_{j}\right)\right)} e^{U_{\delta_{j}, \xi_{j}}} Z_{0 j}\left[8 \pi \delta_{k} \frac{\partial G_{\lambda}}{\partial\left(\xi_{k}\right)_{l}}\left(x, \xi_{k}\right)+O\left(\lambda^{3} \rho^{3}+\frac{\rho^{3}}{\left|x-\xi_{k}\right|^{3}}\right)\right] d x+O\left(\lambda^{3} \rho^{3}\right) \\
& =O\left(\lambda \rho \int_{B\left(\xi_{j}, \varepsilon d\left(\xi_{j}\right)\right)} e^{U_{\delta_{j}, \xi_{j}}} Z_{0 j} d x\right)+O\left(\lambda^{3} \rho^{3}\right) \\
& =O(\lambda \rho),
\end{aligned}
$$

because $\left|\partial_{\left(\xi_{j}\right)_{i}} H_{\lambda}\left(x, \xi_{j}\right)\right|=O(\lambda)$ holds uniformly in $\Omega$, which can be directly proved by (2.15) and the maximum principle with Robin boundary condition.

Now, for $i=1,2$ and $j=1, \ldots, m$, multiplying (3.2) by $P Z_{i j}$ and integrating by parts we find

$$
\sum_{l=1}^{2} \sum_{k=1}^{m} c_{l k} \int_{\Omega} e^{U_{\delta_{k}}, \xi_{k}} Z_{l k} P Z_{i j}+\int_{\Omega} h P Z_{i j}=\int_{\Omega} W_{\xi} \phi P Z_{i j}-\int_{\Omega} e^{U_{\delta_{j}, \xi_{j}}} Z_{i j} \phi .
$$


Since $\left|\int_{\Omega} h P Z_{i j}\right|=O(\|h\| *)$, by (3.23)-(3.24),

$$
\sum_{l=1}^{2} \sum_{k=1}^{m} c_{l k} \int_{\Omega} e^{U_{\delta_{k}, \xi_{k}}} Z_{l k} P Z_{i j}+\int_{\Omega} h P Z_{i j}=D c_{i j}+O\left(e^{-p / 4} \sum_{l=1}^{2} \sum_{k=1}^{m}\left|c_{l k}\right|\right)+O\left(\|h\|_{\star}\right)
$$

where $D=64 \int_{\mathbb{R}^{2}} \frac{|y|^{2}}{\left(1+|y|^{2}\right)^{4}}$. Moreover, by Lemma 3.1,

$$
\begin{aligned}
D c_{i j}+O\left(e^{-p / 4} \sum_{l=1}^{2} \sum_{k=1}^{m}\left|c_{l k}\right|\right)+O\left(\|h\|_{\star}\right) & =\int_{B\left(\xi_{j}, \varepsilon d\left(\xi_{j}\right) \sqrt{\delta_{j}}\right)} W_{\xi} \phi P Z_{i j}-\int_{\Omega} e^{U_{\delta_{j}, \xi_{j}}} Z_{i j} \phi+O\left(\lambda \sqrt{\rho}\|\phi\|_{\infty}\right) \\
& =\int_{B\left(\xi_{j}, \varepsilon d\left(\xi_{j}\right) \sqrt{\delta_{j}}\right)}\left(W_{\xi}-e^{\left.U_{\delta_{j}, \xi_{j}}\right)} \phi P Z_{i j}+\int_{\Omega} e^{U_{\delta_{j}, \xi_{j}}\left(P Z_{i j}-Z_{i j}\right) \phi+O\left(\lambda \sqrt{\rho}\|\phi\|_{\infty}\right)}\right. \\
& =\frac{1}{p} \int_{B\left(0, \varepsilon d\left(\xi_{j}\right) / \sqrt{\left.\delta_{j}\right)}\right.} \frac{32 y_{i}}{\left(1+|y|^{2}\right)^{3}}\left(\omega_{1}-U_{1,0}-\frac{1}{2} U_{1,0}^{2}\right) \widehat{\phi}_{j}(y)+O\left(\frac{1}{p^{2}}\|\phi\|_{\infty}\right),
\end{aligned}
$$

in view of (3.21)-(3.22), where $\widehat{\phi}_{j}(y)=\phi\left(\delta_{j} y+\xi_{j}\right)$. Accordingly, (3.25) can be reduced to

$$
D c_{i j}+O\left(e^{-p / 4} \sum_{l=1}^{2} \sum_{k=1}^{m}\left|c_{l k}\right|\right)=O\left(\|h\|_{\star}+\frac{1}{p}\|\phi\|_{\infty}\right)
$$

for each $i=1,2$ and $j=1, \ldots, m$. Therefore, we obtain

$$
\sum_{l=1}^{2} \sum_{k=1}^{m}\left|c_{l k}\right|=O\left(\|h\|_{\star}+\frac{1}{p}\|\phi\|_{\infty}\right)=o(1) .
$$

As in Step 4, we conclude that for each $j=1, \ldots, m$,

$$
\widehat{\phi}_{j} \rightarrow C_{j} \frac{|y|^{2}-1}{|y|^{2}+1} \text { in } C_{\mathrm{loc}}^{0}\left(\mathbb{R}^{2}\right)
$$

for some constant $C_{j} \in \mathbb{R}$, and thus

$$
\int_{B\left(0, \varepsilon d\left(\xi_{j}\right) / \sqrt{\delta_{j}}\right)} \frac{32 y_{i}}{\left(1+|y|^{2}\right)^{3}}\left(\omega_{1}-U_{1,0}-\frac{1}{2} U_{1,0}^{2}\right) \widehat{\phi}_{j}(y) \rightarrow C_{j} \int_{\mathbb{R}^{2}} \frac{32 y_{i}\left(|y|^{2}-1\right)}{\left(1+|y|^{2}\right)^{4}}\left(\omega_{1}-U_{1,0}-\frac{1}{2} U_{1,0}^{2}\right)(y)=0 .
$$

Therefore,

$$
\sum_{l=1}^{2} \sum_{k=1}^{m}\left|c_{l k}\right|=o\left(\frac{1}{p}\right)+O\left(\|h\|_{\star}\right)
$$

which is impossible because of (3.20).

Step 6:

We prove the solvability of problem (3.2). To this purpose, we consider the spaces

$$
K_{\xi}=\left\{\sum_{i=1}^{2} \sum_{j=1}^{m} c_{i j} P Z_{i j}: c_{i j} \in \mathbb{R} \text { for } i=1,2, j=1, \ldots, m\right\}
$$

and

$$
K_{\xi}^{\perp}=\left\{\phi \in L^{2}(\Omega): \int_{\Omega} e^{U_{\delta_{j}, \xi_{j}}} Z_{i j} \phi=0 \text { for } i=1,2, j=1, \ldots, m\right\} .
$$


Define $\Pi_{\xi}: L^{2}(\Omega) \rightarrow K_{\xi}$ by

$$
\Pi_{\xi} \phi=\sum_{i=1}^{2} \sum_{j=1}^{m} c_{i j} P Z_{i j}
$$

where the coefficients $c_{i j}$ are uniquely determined (as it follows by (3.23)-(3.24)) by the system

$$
\int_{\Omega} e^{U_{\delta_{k}, \xi_{k}}} Z_{l k}\left(\phi-\sum_{i=1}^{2} \sum_{j=1}^{m} c_{i j} P Z_{i j}\right)=0 \quad \text { for any } l=1,2, k=1, \ldots, m .
$$

Let $\Pi_{\xi}^{\perp}=\mathrm{Id}-\Pi_{\xi}: L^{2}(\Omega) \rightarrow K_{\xi}^{\perp}$. Moreover, the Hilbert space $K_{\xi}^{\perp} \cap H^{1}(\Omega)$ is endowed with the inner product

$$
\langle\phi, \psi\rangle_{H}=\int_{\Omega} \nabla \phi \nabla \psi+\lambda \int_{\partial \Omega} b(x) \phi \psi .
$$

Problem (3.2), expressed in a weak form, is equivalent to finding $\phi \in K_{\xi}^{\perp} \cap H^{1}(\Omega)$ such that

$$
\langle\phi, \psi\rangle_{H}=\int_{\Omega}\left(W_{\xi} \phi-h\right) \psi \quad \text { for all } \psi \in K_{\xi}^{\perp} \cap H^{1}(\Omega) .
$$

With the aid of Riesz's theorem, this equation gets rewritten in $K_{\xi}^{\perp} \cap H^{1}(\Omega)$ in the operator form

$$
\phi=K(\phi)+\tilde{h},
$$

where

$$
\tilde{h}=-\Pi_{\xi}^{\perp}\left[\left.(-\Delta)\right|_{\Omega}+\left.\left(\frac{\partial}{\partial v}+\lambda b(x)\right)\right|_{\partial \Omega}\right]^{-1} h \text { and } K(\phi)=\Pi_{\xi}^{\perp}\left[\left.(-\Delta)\right|_{\Omega}+\left.\left(\frac{\partial}{\partial v}+\lambda b(x)\right)\right|_{\partial \Omega}\right]^{-1}\left(W_{\xi} \phi\right)
$$

is a linear compact operator in $K_{\xi}^{\perp} \cap H^{1}(\Omega)$. By Fredholm's alternative with Robin boundary condition (see $[6,14]$ and references therein), we obtain the unique solvability of this problem for any $\tilde{h} \in K_{\xi}^{\perp}$ provided that the homogeneous equation $\phi=K(\phi)$ has only the trivial solution in $K_{\xi}^{\perp} \cap H^{1}(\Omega)$, which in turn follows from the a priori estimate (3.19) in Step 5. Moreover, by the elliptic regularity theory the solution constructed in this way belongs to $H^{2}(\Omega)$. For $p>p_{0}$ and $\lambda>\lambda_{0}$ fixed and under the restrained growth condition (2.4), and by the density of $C^{0, \alpha}(\bar{\Omega})$ in $\left(C(\bar{\Omega}),\|\cdot\|_{\infty}\right)$, we can approximate $h \in C(\bar{\Omega})$ by smooth functions and, by (3.19) and the elliptic regularity theory, we can show that (3.4) holds for any $h \in C(\bar{\Omega})$. This completes the proof.

Remark 3.4. Given $h \in C(\bar{\Omega})$, let $\phi$ be the solution of problem (3.2) given by Proposition 3.3. Multiplying (3.2) against $\phi$ and integrating by parts, we get

$$
\|\phi\|_{H}^{2}:=\int_{\Omega}|\nabla \phi|^{2}+\lambda \int_{\partial \Omega} b(x) \phi^{2}=\int_{\Omega} W_{\xi} \phi^{2}-\int_{\Omega} h \phi .
$$

By Lemma 3.1, we can prove that $\left|\int_{\Omega} W_{\xi} \phi^{2}\right| \leq C\|\phi\|_{\infty}^{2}$, and therefore $\|\phi\|_{H} \leq C\left(\|h\|_{\star}+\|\phi\|_{\infty}\right)$.

\section{The nonlinear problem}

In what follows we want to solve the nonlinear auxiliary problem: for any points $\xi=\left(\xi_{1}, \ldots, \xi_{m}\right) \in \mathcal{O}_{\varepsilon}$, we look for a function $\phi \in H^{2}(\Omega)$ such that

$$
\begin{cases}\Delta\left(U_{\xi}+\phi\right)+\left(U_{\xi}+\phi\right)^{p}=\sum_{i=1}^{2} \sum_{j=1}^{m} c_{i j} e^{U_{\delta_{j}, \xi_{j}}} Z_{i j} & \text { in } \Omega, \\ U_{\xi}+\phi>0 & \text { in } \Omega, \\ \frac{\partial \phi}{\partial v}+\lambda b(x) \phi=0 & \text { on } \partial \Omega, \\ \int_{\Omega} e^{U_{\delta_{j}, \xi_{j}}} Z_{i j} \phi=0 & \text { for } i=1,2, j=1, \ldots, m,\end{cases}
$$


for some coefficients $c_{i j}, i=1,2, j=1, \ldots, m$, which depend on $\xi$. Recalling that

$$
N(\phi)=\left(U_{\xi}+\phi\right)_{+}^{p}-U_{\xi}^{p}-p U_{\xi}^{p-1} \phi \text { and } R_{\xi}=\Delta U_{\xi}+U_{\xi}^{p},
$$

we can rewrite the first equation in (4.1) in the form

$$
L(\phi)=-\left[R_{\xi}+N(\phi)\right]+\sum_{i=1}^{2} \sum_{j=1}^{m} c_{i j} e^{U_{\delta_{j}, \xi_{j}}} Z_{i j} .
$$

Using the theory developed in the previous section for the linear operator $L$, we have the following result.

Proposition 4.1. Let $\varepsilon>0$ be fixed and small. There exist $C>0, p_{0}>0$ and $\lambda_{0}>0$ such that for any points $\xi=\left(\xi_{1}, \ldots, \xi_{m}\right) \in \mathcal{O}_{\varepsilon}$, and any $p>p_{0}$ and $\lambda>\lambda_{0}$ satisfying (2.4), problem (4.1) has a unique solution $\phi_{\xi}$ for some scalars $c_{i j}(\xi), i=1,2, j=1, \ldots, m$, such that

$$
\left\|\phi_{\xi}\right\|_{\infty} \leq \frac{C}{p^{3}}, \quad \sum_{i=1}^{2} \sum_{j=1}^{m}\left|c_{i j}(\xi)\right| \leq \frac{C}{p^{4}} \text { and }\left\|\phi_{\xi}\right\|_{H} \leq \frac{C}{p^{3}} .
$$

Furthermore, the map $\xi \rightarrow \phi_{\xi}$ is a $C^{1}$-function in $C(\bar{\Omega})$ and $H^{1}(\Omega)$.

Proof. The proof of this proposition can be done along the lines of the proof of [13, Lemma 4.1]. We omit the details.

\section{Variational reduction}

After problem (4.1) has been solved, we find a solution of problem (2.22) and hence to the original problem (1.1) if $\xi=\left(\xi_{1}, \ldots, \xi_{m}\right) \in \mathcal{O}_{\varepsilon}$ satisfies

$$
c_{i j}(\xi)=0 \quad \text { for all } i=1,2, j=1, \ldots, m .
$$

Equation (1.1) is the Euler-Lagrange equation of the functional $J_{p}^{\lambda}: H^{1}(\Omega) \rightarrow \mathbb{R}$, defined by

$$
J_{p}^{\lambda}(u)=\frac{1}{2} \int_{\Omega}|\nabla u|^{2}-\frac{1}{p+1} \int_{\Omega} u_{+}^{p+1}+\frac{\lambda}{2} \int_{\partial \Omega} b(x) u^{2} .
$$

We define

$$
F_{p}^{\lambda}(\xi)=J_{p}^{\lambda}\left(U_{\xi}+\phi_{\xi}\right) \quad \text { for all } \xi \in \mathcal{O}_{\varepsilon},
$$

where $\phi_{\xi}$ is the unique solution to problem (4.1) given by Proposition 4.1. The critical points of $F_{p}^{\lambda}$ correspond to solutions of (5.1) for all sufficiently large $p$ and $\lambda$ satisfying (2.4), as the following result states.

Proposition 5.1. The function $F_{p}^{\lambda}: \mathcal{O}_{\varepsilon} \rightarrow \mathbb{R}$ is of class $C^{1}$. Moreover, for all sufficiently large $p$ and $\lambda$ satisfying (2.4), if $D_{\xi} F_{p}^{\lambda}(\xi)=0$, then $\xi$ satisfies (5.1).

Proof. Since the map $\xi \rightarrow \phi_{\xi}$ is a $C^{1}$-map into $H^{1}(\Omega), F_{p}^{\lambda}$ is a $C^{1}$-function of $\xi$. Suppose now that $\xi \in \mathcal{O}_{\varepsilon}$ is a point such that $D_{\xi} F_{p}^{\lambda}(\xi)=0$. Then, we have that

$$
\begin{aligned}
0 & =\int_{\Omega} \nabla\left(U_{\xi}+\phi_{\xi}\right) \nabla\left[D_{\xi}\left(U_{\xi}+\phi_{\xi}\right)\right]-\int_{\Omega}\left(U_{\xi}+\phi_{\xi}\right)^{p} D_{\xi}\left(U_{\xi}+\phi_{\xi}\right)+\lambda \int_{\partial \Omega} b(x)\left(U_{\xi}+\phi_{\xi}\right) D_{\xi}\left(U_{\xi}+\phi_{\xi}\right) \\
& =-\int_{\Omega}\left[\Delta\left(U_{\xi}+\phi_{\xi}\right)+\left(U_{\xi}+\phi_{\xi}\right)^{p}\right] D_{\xi}\left(U_{\xi}+\phi_{\xi}\right) \\
& =-\sum_{i=1}^{2} \sum_{j=1}^{m} c_{i j}(\xi) \int_{\Omega} e^{U_{\delta_{j}, \xi_{j}}} Z_{i j} D_{\xi}\left(U_{\xi}+\phi_{\xi}\right) \\
& =-\sum_{i=1}^{2} \sum_{j=1}^{m} c_{i j}(\xi) \int_{\Omega} e^{U_{\delta_{j}, \xi_{j}}} Z_{i j} D_{\xi} U_{\xi}+\sum_{i=1}^{2} \sum_{j=1}^{m} c_{i j}(\xi) \int_{\Omega} D_{\xi}\left(e^{U_{\delta_{j}, \xi_{j}}} Z_{i j}\right) \phi_{\xi},
\end{aligned}
$$


because $\int_{\Omega} e^{U_{\delta_{j}, \xi_{j}}} Z_{i j} \phi_{\xi}=0$. From the definition of $U_{\xi}$ in (2.12), we obtain

$$
\begin{aligned}
\partial_{\left(\xi_{k}\right)_{l}} U_{\xi}= & \sum_{j=1}^{m} \frac{1}{y \mu_{j}^{2 /(p-1)}}\left\{\partial_{\left(\xi_{k}\right)_{l}}\left[U_{\delta_{j}, \xi_{j}}(x)+\frac{1}{p} \omega_{1}\left(\frac{x-\xi_{j}}{\delta_{j}}\right)+\frac{1}{p^{2}} \omega_{2}\left(\frac{x-\xi_{j}}{\delta_{j}}\right)+y \mu_{j}^{2 /(p-1)} H_{j}(x)\right]\right. \\
& \left.-\frac{2 \partial_{\left(\xi_{k}\right)_{l} l} \log \mu_{j}}{p-1}\left[U_{\delta_{j}, \xi_{j}}(x)+\frac{1}{p} \omega_{1}\left(\frac{x-\xi_{j}}{\delta_{j}}\right)+\frac{1}{p^{2}} \omega_{2}\left(\frac{x-\xi_{j}}{\delta_{j}}\right)+y \mu_{j}^{2 /(p-1)} H_{j}(x)\right]\right\} \\
= & \sum_{j=1}^{m} \frac{1}{y \mu_{j}^{2 /(p-1)}}\left\{\partial_{\left(\xi_{k}\right)_{l}}\left[U_{\delta_{j}, \xi_{j}}(x)+\frac{1}{p} \omega_{1}\left(\frac{x-\xi_{j}}{\delta_{j}}\right)+\frac{1}{p^{2}} \omega_{2}\left(\frac{x-\xi_{j}}{\delta_{j}}\right)+y \mu_{j}^{2 /(p-1)} H_{j}(x)\right]\right. \\
& \left.-\frac{2 \partial_{\left(\xi_{k}\right)_{l}} \log \mu_{j}}{p-1}[p+O(1)]\right\} .
\end{aligned}
$$

Notice that by (2.1) and (3.3),

$$
\partial_{\left(\xi_{k}\right)_{l}} U_{\delta_{j}, \xi_{j}}(x)=\frac{1}{\delta_{j}} \delta_{k j} Z_{l j}+2 Z_{0 j} \partial_{\left(\xi_{k}\right)_{l}} \log \mu_{j}=\frac{1}{\delta_{j}} \delta_{k j} Z_{l j}+2\left(1-\frac{2 \delta_{j}^{2}}{\left|x-\xi_{j}\right|^{2}+\delta_{j}^{2}}\right) \partial_{\left(\xi_{k}\right)_{l}} \log \mu_{j},
$$

while for $i=1$, 2, by (2.9) and (3.3),

$$
\begin{aligned}
\partial_{\left(\xi_{k}\right)_{l}} \omega_{i}\left(\frac{x-\xi_{j}}{\delta_{j}}\right) & =-\omega_{i}^{\prime}\left(\frac{\left|x-\xi_{j}\right|}{\delta_{j}}\right)\left\{\frac{\left(x-\xi_{j}\right)_{l}}{\left|x-\xi_{j}\right|} \frac{1}{\delta_{j}} \delta_{k j}+\frac{\left|x-\xi_{j}\right|}{\delta_{j}} \partial_{\left(\xi_{k}\right)_{l}} \log \mu_{j}\right\} \\
& =-\frac{1}{\delta_{j}} \delta_{k j}\left[\frac{C_{i}}{4} Z_{l j}+O\left(\frac{\delta_{j}^{2}}{\left|x-\xi_{j}\right|^{2}+\delta_{j}^{2}}\right)\right]-C_{i}\left[1+O\left(\frac{\delta_{j}^{2}}{\left|x-\xi_{j}\right|^{2}+\delta_{j}^{2}}\right)\right] \partial_{\left(\xi_{k}\right)_{l}} \log \mu_{j},
\end{aligned}
$$

where $\delta_{k j}$ denotes Kronecker's symbol. Moreover, as in the proof of Lemma 2.1, we can prove that

$$
\begin{aligned}
\partial_{\left(\xi_{k}\right) l}\left[y \mu_{j}^{2 /(p-1)} H_{j}(x)\right]= & \delta_{k j}\left(1-\frac{C_{1}}{4 p}-\frac{C_{2}}{4 p^{2}}\right) 8 \pi \partial_{\left(\xi_{k}\right)_{l}} H_{\lambda}\left(x, \xi_{j}\right)+\left(-2+\frac{C_{1}}{p}+\frac{C_{2}}{p^{2}}\right) \partial_{\left(\xi_{k}\right)_{l}} \log \mu_{j} \\
& +\frac{1}{p} O\left(\frac{\rho}{d\left(\xi_{j}\right)^{3}}+\frac{\rho}{d\left(\xi_{j}\right)^{2}}\left|\partial_{\left(\xi_{k}\right)_{l}} \log \mu_{j}\right|\right) .
\end{aligned}
$$

Furthermore, by (2.3)-(2.5), (2.19) and (3.21), we get

$$
\begin{aligned}
\partial_{\left(\xi_{k}\right)_{l}} U_{\xi}= & \frac{1}{\delta_{k} y \mu_{k}^{2 /(p-1)}}\left\{Z_{l k}-\frac{1}{p} \omega_{1}^{\prime}\left(\frac{\left|x-\xi_{k}\right|}{\delta_{k}}\right) \frac{\left(x-\xi_{k}\right)_{l}}{\left|x-\xi_{k}\right|}-\frac{1}{p^{2}} \omega_{2}^{\prime}\left(\frac{\left|x-\xi_{k}\right|}{\delta_{k}}\right) \frac{\left(x-\xi_{k}\right)_{l}}{\left|x-\xi_{k}\right|}\right. \\
& \left.+\left(1-\frac{C_{1}}{4 p}-\frac{C_{2}}{4 p^{2}}\right) 8 \pi \delta_{k} \partial_{\left(\xi_{k}\right)_{l}} H_{\lambda}\left(x, \xi_{k}\right)\right\} \\
& +\sum_{j=1}^{m} \frac{1}{y \mu_{j}^{2 /(p-1)}}\left\{\left[-2+O\left(\frac{1}{p}+\frac{\delta_{j}^{2}}{\left|x-\xi_{j}\right|^{2}+\delta_{j}^{2}}\right)\right] \partial_{\left(\xi_{k}\right)_{l}} \log \mu_{j}+O\left(\frac{\lambda^{3} \rho}{p}\right)\right\} \\
= & \frac{1}{\delta_{k} y \mu_{k}^{2 /(p-1)}}\left\{\left(1-\frac{C_{1}}{4 p}-\frac{C_{2}}{4 p^{2}}\right) P Z_{l k}+O\left(\lambda^{3} \rho^{3}+\frac{1}{p} \frac{\delta_{k}^{2}}{\left|x-\xi_{k}\right|^{2}+\delta_{k}^{2}}\right)\right\}+O\left(\frac{\sqrt{\lambda}}{y}\right) .
\end{aligned}
$$

On the other hand, by (2.1) and (3.3), for $i=1,2$, we have that

$$
\partial_{\left(\xi_{k}\right)_{l}}\left(e^{U_{\delta_{j}, \xi_{j}}} Z_{i j}\right)=-4 \delta_{j} e^{U_{\delta_{j}, \xi_{j}}}\left[\frac{\delta_{i l}}{\left|x-\xi_{j}\right|^{2}+\delta_{j}^{2}}-6 \frac{\left(x-\xi_{j}\right)_{i}\left(x-\xi_{k}\right)_{l}}{\left(\left|x-\xi_{j}\right|^{2}+\delta_{j}^{2}\right)^{2}}\right] \delta_{j k}+3 e^{U_{\delta_{j}, \xi_{j}}} Z_{0 j} Z_{i j} \partial_{\left(\xi_{k}\right)_{l}} \log \mu_{j} .
$$

Consequently, for each $l=1,2$ and $k=1, \ldots, m,(5.4)$ can be written as

$$
-\sum_{i, j} \frac{c_{i j}(\xi)\left[1+O\left(\frac{1}{p}\right)\right]}{\delta_{k} y \mu_{k}^{2 /(p-1)}} \int_{\Omega} e^{U_{\delta_{j}, \xi_{j}}} Z_{i j} P Z_{l k}+\sum_{i, j}\left|c_{i j}(\xi)\right| O\left(\frac{\sqrt{\lambda}}{y}+\left\|\phi_{\xi}\right\|_{\infty} \int_{\Omega}\left|\partial_{\left(\xi_{k}\right)_{l}}\left(e^{U_{\delta_{j}, r_{j}}} Z_{i j}\right)\right|\right)=0,
$$


so that, using (2.19), (3.23), (4.2) and (5.6),

$$
-\frac{64 c_{l k}(\xi)}{\delta_{k} y \mu_{k}^{2 /(p-1)}} \int_{\mathbb{R}^{2}} \frac{|y|^{2}}{\left(1+|y|^{2}\right)^{4}} d y+O\left(\frac{1}{\delta_{k} p y}+\frac{\lambda}{y}+\frac{1}{p^{3} \delta_{k}}+\frac{\sqrt{\lambda}}{p^{3}}\right) \sum_{i=1}^{2} \sum_{j=1}^{m}\left|c_{i j}(\xi)\right|=0,
$$

which, together with (2.5), implies $c_{l k}(\xi)=0$ for each $l=1,2$ and $k=1, \ldots, m$.

Next, we need to write the expansion of $F_{p}^{\lambda}(\xi)$ in terms of $\varphi_{m}^{\lambda}(\xi)$ as $p$ and $\lambda$ go to $+\infty$ and satisfy (2.4).

Proposition 5.2. Let $\varepsilon>0$ be fixed and small. With the choice (2.17) for the parameters $\mu_{j}$, there exist $p_{0}>0$ and $\lambda_{0}>0$ such that for any points $\xi=\left(\xi_{1}, \ldots, \xi_{m}\right) \in \mathcal{O}_{\varepsilon}$, and any $p>p_{0}$ and $\lambda>\lambda_{0}$ satisfying (2.4), the following expansion holds uniformly:

$$
F_{p}^{\lambda}(\xi)=\frac{4 \pi m p}{y^{2}}-\frac{16 \pi m \log \lambda}{y^{2}}-\frac{32 \pi^{2}}{y^{2}} \varphi_{m}^{\lambda}(\xi)+\frac{4 \pi m}{y^{2}}+\frac{m}{2 y^{2}} \int_{\mathbb{R}^{2}}\left(\frac{8}{\left(1+|y|^{2}\right)^{2}} U_{1,0}-\Delta \omega_{1}\right)+O\left(\frac{\log \lambda}{p^{3}}\right) .
$$

Proof. Multiplying the first equation in (4.1) by $U_{\xi}+\phi_{\xi}$ and integrating by parts, we obtain

$$
\int_{\Omega}\left(U_{\xi}+\phi_{\xi}\right)^{p+1}=\int_{\Omega}\left|\nabla\left(U_{\xi}+\phi_{\xi}\right)\right|^{2}+\lambda \int_{\partial \Omega} b(x)\left(U_{\xi}+\phi_{\xi}\right)^{2}+\sum_{i=1}^{2} \sum_{j=1}^{m} c_{i j}(\xi) \int_{\Omega} e^{U_{\delta_{j}, \xi_{j}}} Z_{i j} U_{\xi} .
$$

Since $U_{\xi}$ is a bounded function, by (4.2) we get

$$
\int_{\Omega}\left(U_{\xi}+\phi_{\xi}\right)^{p+1}=\int_{\Omega}\left|\nabla\left(U_{\xi}+\phi_{\xi}\right)\right|^{2}+\lambda \int_{\partial \Omega} b(x)\left(U_{\xi}+\phi_{\xi}\right)^{2}+O\left(\frac{1}{p^{4}}\right)
$$

uniformly for any points $\xi=\left(\xi_{1}, \ldots, \xi_{m}\right) \in \mathcal{O}_{\varepsilon}$. Hence, by (5.2)-(5.3) we have

$$
\begin{aligned}
F_{p}^{\lambda}(\xi)= & \left(\frac{1}{2}-\frac{1}{p+1}\right)\left[\int_{\Omega}\left|\nabla\left(U_{\xi}+\phi_{\xi}\right)\right|^{2}+\lambda \int_{\partial \Omega} b(x)\left(U_{\xi}+\phi_{\xi}\right)^{2}\right]+O\left(\frac{1}{p^{4}}\right) \\
= & \left(\frac{1}{2}-\frac{1}{p+1}\right)\left[\left(\int_{\Omega}\left|\nabla U_{\xi}\right|^{2}+\lambda \int_{\partial \Omega} b(x) U_{\xi}^{2}\right)+2\left(\int_{\Omega} \nabla U_{\xi} \nabla \phi_{\xi}+\lambda \int_{\partial \Omega} b(x) U_{\xi} \phi_{\xi}\right)\right. \\
& \left.+\left(\int_{\Omega}\left|\nabla \phi_{\xi}\right|^{2}+\lambda \int_{\partial \Omega} b(x) \phi_{\xi}^{2}\right)\right]+O\left(\frac{1}{p^{4}}\right) .
\end{aligned}
$$

In view of (2.16), (2.20) and (2.25) we have

$$
\begin{aligned}
\int_{\Omega}\left|\nabla U_{\xi}\right|^{2}+\lambda \int_{\partial \Omega} b(x) U_{\xi}^{2}= & \int_{\Omega}\left(-\Delta U_{\xi}\right) U_{\xi} \\
= & \sum_{j=1}^{m} \frac{1}{y \mu_{j}^{2 /(p-1)}} \int_{B\left(\xi_{j}, \varepsilon d\left(\xi_{j}\right)\right)}\left[e^{U_{\delta_{j}, \xi_{j}}}-\frac{1}{p \delta_{j}^{2}} \Delta \omega_{1}\left(\frac{x-\xi_{j}}{\delta_{j}}\right)-\frac{1}{p^{2} \delta_{j}^{2}} \Delta \omega_{2}\left(\frac{x-\xi_{j}}{\delta_{j}}\right)\right] U_{\xi} \\
& +O\left(\rho^{2} \lambda^{2} \log \lambda\right) \\
= & \sum_{j=1}^{m} \frac{1}{y^{2} \mu_{j}^{4 /(p-1)}} \int_{B\left(0, \varepsilon d\left(\xi_{j}\right) / \delta_{j}\right)}\left[\frac{8}{\left(1+|y|^{2}\right)^{2}}-\frac{1}{p} \Delta \omega_{1}(y)-\frac{1}{p^{2}} \Delta \omega_{2}(y)\right] \\
& \times\left[p+U_{1,0}(y)+\frac{1}{p} \omega_{1}(y)+\frac{1}{p^{2}} \omega_{2}(y)+O(\lambda \rho|y|)+\frac{1}{p} O(\lambda \rho)\right] d y+O\left(\rho^{2} \lambda^{2} \log \lambda\right) \\
= & \sum_{j=1}^{m} \frac{1}{y^{2} \mu_{j}^{4 /(p-1)}}\left[8 \pi p+\int_{\mathbb{R}^{2}}\left(\frac{8}{\left(1+|y|^{2}\right)^{2}} U_{1,0}-\Delta \omega_{1}\right)+O\left(\frac{1}{p}\right)\right] \\
= & \frac{8 \pi m p}{y^{2}}-\frac{32 \pi}{y^{2}} \sum_{j=1}^{m} \log \mu_{j}+\frac{m}{y^{2}} \int_{\mathbb{R}^{2}}\left(\frac{8}{\left(1+|y|^{2}\right)^{2}} U_{1,0}-\Delta \omega_{1}\right)+O\left(\frac{1}{p^{3}}\right),
\end{aligned}
$$


since $\mu_{j}^{-4 /(p-1)}=1-\frac{4}{p} \log \mu_{j}+O\left(\frac{1}{p^{2}}\right)$. Recalling the expansion (2.17) of $\mu_{j}$ and the definition (1.9) of $\varphi_{m}^{\lambda}(\xi)$, we get

$$
\begin{aligned}
\int_{\Omega}\left|\nabla U_{\xi}\right|^{2}+\lambda \int_{\partial \Omega} b(x) U_{\xi}^{2}= & \frac{8 \pi m p}{y^{2}}-\frac{32 \pi m \log \lambda}{y^{2}}-\frac{64 \pi^{2}}{y^{2}} \varphi_{m}^{\lambda}(\xi) \\
& +\frac{24 \pi m}{y^{2}}+\frac{m}{y^{2}} \int_{\mathbb{R}^{2}}\left(\frac{8}{\left(1+|y|^{2}\right)^{2}} U_{1,0}-\Delta \omega_{1}\right)+O\left(\frac{\log \lambda}{p^{3}}\right) .
\end{aligned}
$$

On the other hand, by virtue of (4.2), we have

$$
2\left(\int_{\Omega} \nabla U_{\xi} \nabla \phi_{\xi}+\lambda \int_{\partial \Omega} b(x) U_{\xi} \phi_{\xi}\right)+\left(\int_{\Omega}\left|\nabla \phi_{\xi}\right|^{2}+\lambda \int_{\partial \Omega} b(x) \phi_{\xi}^{2}\right)=O\left(\frac{1}{p^{7 / 2}}\right) .
$$

Consequently, inserting (5.9) and (5.10) in (5.8), we obtain (5.7).

Finally, we want to show that the expansion of $F_{p}^{\lambda}(\xi)$ in terms of $\varphi_{m}^{\lambda}(\xi)$ holds in a $C^{1}$-sense.

Proposition 5.3. Let $\varepsilon>0$ be fixed and small. Then, there exist $p_{0}>0$ and $\lambda_{0}>0$ such that for any points $\xi=\left(\xi_{1}, \ldots, \xi_{m}\right) \in \mathcal{O}_{\varepsilon}$, and any $p>p_{0}$ and $\lambda>\lambda_{0}$ satisfying (2.4), the following expansion holds uniformly:

$$
\nabla_{\left(\xi_{k}\right)_{l}} F_{p}^{\lambda}(\xi)=-\frac{32 \pi^{2}}{y^{2}} \nabla_{\left(\xi_{k}\right)_{l}} \varphi_{m}^{\lambda}\left(\xi_{1}, \ldots, \xi_{m}\right)+O\left(\frac{\lambda}{p^{5}}\right)+O\left(\frac{1}{p^{3}}\right)
$$

where $l=1,2$ and $k=1, \ldots, m$.

Proof. Observe that for any $l=1,2$ and $k=1, \ldots, m$,

$$
\partial_{\left(\xi_{k}\right)_{l}} F_{p}^{\lambda}(\xi)=-\int_{\Omega}\left(\Delta u_{\xi}+u_{\xi}^{p}\right) \partial_{\left(\xi_{k}\right)_{l}} U_{\xi}-\int_{\Omega}\left(\Delta u_{\xi}+u_{\xi}^{p}\right) \partial_{\left(\xi_{k}\right)_{l}} \phi_{\xi},
$$

where $u_{\xi}=U_{\xi}+\phi_{\xi}$. For each $k=1, \ldots, m$, let $\eta_{k}$ be a radial smooth cut-off function such that $0 \leq \eta_{k} \leq 1$, $\eta_{k}=1$ if $|x| \leq \varepsilon d\left(\xi_{k}\right)$, and $\eta_{k}=0$ if $|x| \geq 2 \varepsilon d\left(\xi_{k}\right)$. In view of (4.1), we deduce

$$
\begin{aligned}
\int_{\Omega}\left(\Delta u_{\xi}+u_{\xi}^{p}\right) \partial_{\left(\xi_{k}\right)_{l}} \phi_{\xi}= & \sum_{i=1}^{2} \sum_{j=1}^{m} c_{i j}(\xi) \int_{\Omega} e^{U_{\delta_{j}, \xi_{j}}} Z_{i j} \partial_{\left(\xi_{k}\right)_{l}} \phi_{\xi} \\
= & -\sum_{i=1}^{2} \sum_{j=1}^{m} c_{i j}(\xi) \int_{\Omega} \partial_{\left(\xi_{k}\right)_{l}}\left(e^{U_{\delta_{j}, \xi_{j}}} Z_{i j}\right) \phi_{\xi} \\
= & \sum_{i=1}^{2} \sum_{j=1}^{m} c_{i j}(\xi) \int_{\Omega} \partial_{x_{l}}\left(e^{U_{\delta_{j}, \xi_{j}}} Z_{i j}\right) \eta_{k}\left(x-\xi_{k}\right) \phi_{\xi} \\
& -\sum_{i=1}^{2} \sum_{j=1}^{m} c_{i j}(\xi) \int_{\Omega}\left[\partial_{\left(\xi_{k}\right)_{l}}\left(e^{U_{\delta_{j}, \xi_{j}}} Z_{i j}\right)+\eta_{k}\left(x-\xi_{k}\right) \partial_{x_{l}}\left(e^{U_{\delta_{j}, \xi_{j}}} Z_{i j}\right)\right] \phi_{\xi} .
\end{aligned}
$$

Observe that $p^{2} \phi_{\xi}(x) \rightarrow 0$ in $C(\bar{\Omega})$ and thus, by the elliptic regularity theory,

$$
p^{2} \phi_{\xi}(x) \rightarrow 0 \quad \text { uniformly in } C_{\text {loc }}^{1}\left(\Omega \backslash \bigcup_{h=1}^{m} B\left(\xi_{h}, \varepsilon d\left(\xi_{h}\right)\right)\right) .
$$

Since $\eta_{k}\left(x-\xi_{k}\right) \equiv 0$ on $\partial \Omega$, using an integration by parts of the derivative in $x_{l}$ and (4.2), we get

$$
\begin{aligned}
\int_{\Omega} \partial_{x_{l}}\left(e^{U_{\delta_{j}, \xi_{j}}} Z_{i j}\right) \eta_{k}\left(x-\xi_{k}\right) \phi_{\xi} & =\int_{\Omega} \partial_{x_{l}}\left(e^{U_{\delta_{j}, \xi_{j}}} Z_{i j} \eta_{k}\left(x-\xi_{k}\right) \phi_{\xi}\right)-\int_{\Omega} e^{U_{\delta_{j}, \xi_{j}}} Z_{i j} \partial_{x_{l}}\left(\eta_{k}\left(x-\xi_{k}\right) \phi_{\xi}\right) \\
& =-\int_{\Omega} e^{U_{\delta_{j}, \xi_{j}}} Z_{i j} \partial_{x_{l}}\left(\eta_{k}\left(x-\xi_{k}\right) \phi_{\xi}\right) \\
& =-\int_{B\left(\xi_{k}, \varepsilon d\left(\xi_{k}\right)\right)} e^{U_{\delta_{j}, \xi_{j}}} Z_{i j} \partial_{x_{l}} \phi_{\xi}+O\left(\frac{\lambda^{4} \rho^{3}}{p^{3}}\right),
\end{aligned}
$$


in view of $\left|\partial_{x_{l}} \eta_{k}\left(x-\xi_{k}\right)\right|=O(\lambda)$. On the other hand, by (5.6) we have

$$
\begin{aligned}
\partial_{\left(\xi_{k}\right)_{l}}\left(e^{U_{\delta_{j}, \xi_{j}}} Z_{i j}\right)+\eta_{k}\left(x-\xi_{k}\right) \partial_{x_{l}}\left(e^{U_{\delta_{j}, \xi_{j}}} Z_{i j}\right) & \\
& =4 \delta_{j} e^{U_{\delta_{j}, \xi_{j}}} \frac{\eta_{k}\left(x-\xi_{k}\right)-\delta_{j k}}{\left|x-\xi_{j}\right|^{2}+\delta_{j}^{2}} \delta_{i l}+24 \delta_{j} e^{U_{\delta_{j}, \xi_{j}}} \frac{\left(x-\xi_{j}\right)_{i}\left(x-\xi_{k}\right)_{l}}{\left(\left|x-\xi_{j}\right|^{2}+\delta_{j}^{2}\right)^{2}}\left(\delta_{j k}-\eta_{k}\left(x-\xi_{k}\right)\right)+3 e^{U_{\delta_{j}, \xi_{j}}} Z_{0 j} Z_{i j} \partial_{\left(\xi_{k}\right)_{l}} \log \mu_{j} \\
& =3 e^{U_{\delta_{j}, \xi_{j}}} Z_{0 j} Z_{i j} \partial_{\left(\xi_{k}\right)_{l}} \log \mu_{j}+O\left(\lambda^{6} \rho^{3}\right),
\end{aligned}
$$

which, together with (2.19), (4.2), and the fact that $\left|Z_{0 j} Z_{i j}\right| \leq 2$, implies

$$
\left|\sum_{i=1}^{2} \sum_{j=1}^{m} c_{i j}(\xi) \int_{\Omega}\left[\partial_{\left(\xi_{k}\right)_{l}}\left(e^{U_{\delta_{j}, \xi_{j}}} Z_{i j}\right)+\eta_{k}\left(x-\xi_{k}\right) \partial_{x_{l}}\left(e^{U_{\delta_{j}}, \xi_{j}} Z_{i j}\right)\right] \phi_{\xi}\right|=O\left(\frac{\sqrt{\lambda}}{p^{7}}\right) .
$$

Therefore,

$$
\begin{aligned}
\int_{\Omega}\left(\Delta u_{\xi}+u_{\xi}^{p}\right) \partial_{\left(\xi_{k}\right) l} \phi_{\xi} & =-\sum_{i=1}^{2} \sum_{j=1}^{m} c_{i j}(\xi) \int_{B\left(\xi_{k}, \varepsilon d\left(\xi_{k}\right)\right)} e^{U_{\delta_{j}, \xi_{j}}} Z_{i j} \partial_{x_{l}} \phi_{\xi}+O\left(\frac{\sqrt{\lambda}}{p^{7}}\right) \\
& =-\int_{B\left(\xi_{k}, \varepsilon d\left(\xi_{k}\right)\right)}\left(\Delta u_{\xi}+u_{\xi}^{p}\right) \partial_{x_{l}} \phi_{\xi}+O\left(\frac{\sqrt{\lambda}}{p^{7}}\right) .
\end{aligned}
$$

Now, by the expression of $U_{\xi}$ in (2.12) we obtain

$$
\partial_{x_{l}} U_{\xi}=\sum_{j=1}^{m} \frac{1}{y \mu_{j}^{2 /(p-1)}} \partial_{x_{l}}\left[U_{\delta_{j}, \zeta_{j}}(x)+\frac{1}{p} \omega_{1}\left(\frac{x-\xi_{j}}{\delta_{j}}\right)+\frac{1}{p^{2}} \omega_{2}\left(\frac{x-\xi_{j}}{\delta_{j}}\right)+y \mu_{j}^{2 /(p-1)} H_{j}(x)\right] .
$$

Obviously, by (2.1) and (3.3),

$$
\partial_{x_{l}} U_{\delta_{j}, \xi_{j}}(x)=-\frac{1}{\delta_{j}} Z_{l j}
$$

while for $i=1$, 2, by (2.9) and (3.3),

$$
\partial_{x_{l}} \omega_{i}\left(\frac{x-\xi_{j}}{\delta_{j}}\right)=\omega_{i}^{\prime}\left(\frac{\left|x-\xi_{j}\right|}{\delta_{j}}\right) \frac{\left(x-\xi_{j}\right)_{l}}{\left|x-\xi_{j}\right|} \frac{1}{\delta_{j}}=\frac{1}{\delta_{j}}\left[\frac{C_{i}}{4} Z_{l j}+O\left(\frac{\delta_{j}^{2}}{\left|x-\xi_{j}\right|^{2}+\delta_{j}^{2}}\right)\right] .
$$

Moreover, as in the proof of Lemma 2.1, for $\left|x-\xi_{k}\right| \leq 2 \varepsilon d\left(\xi_{k}\right)$, we have that

$$
\partial_{x_{l}}\left[y \mu_{j}^{2 /(p-1)} H_{j}(x)\right]=\left(1-\frac{C_{1}}{4 p}-\frac{C_{2}}{4 p^{2}}\right) 8 \pi \partial_{x_{l}} H_{\lambda}\left(x, \xi_{j}\right)+\frac{1}{p} O\left(\frac{\rho}{d\left(\xi_{j}\right) d\left(\xi_{k}\right)}\right) .
$$

Then, for $\left|x-\xi_{k}\right| \leq 2 \varepsilon d\left(\xi_{k}\right)$,

$$
\begin{aligned}
\partial_{x_{l}} U_{\xi}= & \sum_{j=1}^{m} \frac{1}{\delta_{j} y \mu_{j}^{2 /(p-1)}}\left\{-Z_{l j}+\frac{1}{p} \omega_{1}^{\prime}\left(\frac{\left|x-\xi_{j}\right|}{\delta_{j}}\right) \frac{\left(x-\xi_{j}\right)_{l}}{\left|x-\xi_{j}\right|}+\frac{1}{p^{2}} \omega_{2}^{\prime}\left(\frac{\left|x-\xi_{j}\right|}{\delta_{j}}\right) \frac{\left(x-\xi_{j}\right)_{l}}{\left|x-\xi_{j}\right|}\right. \\
& \left.+\left(1-\frac{C_{1}}{4 p}-\frac{C_{2}}{4 p^{2}}\right) 8 \pi \delta_{j} \partial_{x_{l}} H_{\lambda}\left(x, \xi_{j}\right)+\frac{1}{p} O\left(\lambda^{2} \rho^{2}\right)\right\} \\
= & \frac{1}{\delta_{k} y \mu_{k}^{2 /(p-1)}}\left\{-Z_{l k}+\frac{1}{p} \omega_{1}^{\prime}\left(\frac{\left|x-\xi_{k}\right|}{\delta_{k}}\right) \frac{\left(x-\xi_{k}\right)_{l}}{\left|x-\xi_{k}\right|}+\frac{1}{p^{2}} \omega_{2}^{\prime}\left(\frac{\left|x-\xi_{k}\right|}{\delta_{k}}\right) \frac{\left(x-\xi_{k}\right)_{l}}{\left|x-\xi_{k}\right|}\right. \\
& \left.+\left(1-\frac{C_{1}}{4 p}-\frac{C_{2}}{4 p^{2}}\right) 8 \pi \delta_{k} \partial_{x_{l}} H_{\lambda}\left(x, \xi_{k}\right)\right\}+O\left(\frac{1}{y}\right) .
\end{aligned}
$$

Combing (5.5) and (5.14), we find

$$
\partial_{\left(\xi_{k}\right)_{l}} U_{\xi}+\eta_{k}\left(x-\xi_{k}\right) \partial_{x_{l}} U_{\xi}=\frac{8 \pi\left[1+O\left(\frac{1}{p}\right)\right]}{y \mu_{k}^{2 /(p-1)}}\left[\partial_{\left(\xi_{k}\right)_{l}} H_{\lambda}\left(x, \xi_{k}\right)+\eta_{k}\left(x-\xi_{k}\right) \partial_{x_{l}} H_{\lambda}\left(x, \xi_{k}\right)\right]+O\left(\frac{\sqrt{\lambda}}{y}\right)=O\left(\frac{\lambda}{y}\right) .
$$


Furthermore,

$$
\begin{aligned}
\int_{\Omega}\left(\Delta u_{\xi}+u_{\xi}^{p}\right) \partial_{\left(\xi_{k}\right)_{l}} U_{\xi}= & \sum_{i=1}^{2} \sum_{j=1}^{m} c_{i j}(\xi) \int_{\Omega} e^{U_{\delta_{j}, \xi_{j}}} Z_{i j}\left[\partial_{\left(\xi_{k}\right)_{l}} U_{\xi}+\eta_{k}\left(x-\xi_{k}\right) \partial_{x_{l}} U_{\xi}\right] \\
& -\sum_{i=1}^{2} \sum_{j=1}^{m} c_{i j}(\xi) \int_{\Omega} e^{U_{\delta_{j}, \xi_{j}} Z_{i j} \eta_{k}\left(x-\xi_{k}\right) \partial_{x_{l}} U_{\xi}} \\
= & -\sum_{i=1}^{2} \sum_{j=1}^{m} c_{i j}(\xi) \int_{B\left(\xi_{k}, \varepsilon d\left(\xi_{k}\right)\right)} e^{U_{\delta_{j}, \xi_{j}}} Z_{i j} \partial_{x_{l}} U_{\xi}+O\left(\frac{\lambda}{p^{5}}\right) \\
= & -\int_{B\left(\xi_{k}, \varepsilon d\left(\xi_{k}\right)\right)}\left(\Delta u_{\xi}+u_{\xi}^{p}\right) \partial_{x_{l}} U_{\xi}+O\left(\frac{\lambda}{p^{5}}\right) .
\end{aligned}
$$

Inserting (5.13) and (5.15) in (5.11), we get

$$
\partial_{\left(\xi_{k}\right)_{l}} F_{p}^{\lambda}(\xi)=\int_{B\left(\xi_{k}, \varepsilon d\left(\xi_{k}\right)\right)}\left(\Delta u_{\xi}+u_{\xi}^{p}\right) \partial_{x_{l}} u_{\xi}+O\left(\frac{\lambda}{p^{5}}\right) .
$$

Finally, by integrating by parts we have the following Pohozaev-type identities. For any ball $B \subset \Omega$ and for any function $u$,

$$
\int_{B} \Delta u \partial_{x_{l}} u=\int_{\partial B}\left(\partial_{\nu} u \partial_{x_{l}} u-\frac{1}{2}|\nabla u|^{2} v_{l}\right) \text { and } \int_{B} u^{p} \partial_{x_{l}} u=\frac{1}{p+1} \int_{\partial B} u^{p+1} v_{l},
$$

where $v_{l}(x)$ denotes the $l$-component of the exterior unit normal vector $v(x)$ of $\partial B$ at $x \in \partial B$. Let us define

$$
\varphi_{k}(x)=H_{\lambda}\left(x, \xi_{k}\right)+\sum_{j=k} G_{\lambda}\left(x, \xi_{j}\right)
$$

Observe that for sufficiently large $p$ and $\lambda$ satisfying (2.4), by (2.16) and (3.15), we have

$$
y U_{\xi}(x)=8 \pi \sum_{j=1}^{m} G_{\lambda}\left(x, \xi_{j}\right)+O\left(\frac{1}{p}\right)=8 \pi\left(\frac{1}{2 \pi} \log \frac{1}{\left|\chi-\xi_{k}\right|}+\varphi_{k}(x)\right)+O\left(\frac{1}{p}\right)
$$

uniformly in $C_{\text {loc }}^{1}\left(\Omega \backslash \bigcup_{h=1}^{m} B\left(\xi_{h}, \varepsilon d\left(\xi_{h}\right)\right)\right)$. Using (5.12), (5.17), (5.18) and the fact that $u_{\xi}=U_{\xi}+\phi_{\xi}$, we obtain

$$
\begin{aligned}
\int_{B\left(\xi_{k}, \varepsilon d\left(\xi_{k}\right)\right)}\left(\Delta u_{\xi}+u_{\xi}^{p}\right) \partial_{x_{l}} u_{\xi} & =\int_{\partial B\left(\xi_{k}, \varepsilon d\left(\xi_{k}\right)\right)}\left(\partial_{v} u_{\xi} \partial_{x_{l}} u_{\xi}-\frac{1}{2}\left|\nabla u_{\xi}\right|^{2} v_{l}+\frac{1}{p+1} u_{\xi}^{p+1} v_{l}\right) \\
& =\frac{64 \pi^{2}}{y^{2}}\left[\int_{\partial B\left(\xi_{k}, \varepsilon d\left(\xi_{k}\right)\right)}\left(-\frac{1}{2 \pi \varepsilon d\left(\xi_{k}\right)}+\partial_{v} \varphi_{k}\right)\left(-\frac{1}{2 \pi \varepsilon d\left(\xi_{k}\right)} v_{l}+\partial_{x_{l}} \varphi_{k}\right)\right. \\
& \left.-\frac{1}{2} \int_{\partial B\left(\xi_{k}, \varepsilon d\left(\xi_{k}\right)\right)}\left|-\frac{1}{2 \pi \varepsilon d\left(\xi_{k}\right)} v+\nabla \varphi_{k}\right|^{2} v_{l}\right]+O\left(\frac{1}{p^{3}}\right) \\
& =\frac{64 \pi^{2}}{y^{2}} \int_{\partial B\left(\xi_{k}, \varepsilon d\left(\xi_{k}\right)\right)}\left[-\frac{1}{2 \pi \varepsilon d\left(\xi_{k}\right)} \partial_{x_{l}} \varphi_{k}+\left(\partial_{v} \varphi_{k} \partial_{x_{l}} \varphi_{k}-\frac{1}{2}\left|\nabla \varphi_{k}\right|^{2} v_{l}\right)\right]+O\left(\frac{1}{p^{3}}\right) \\
& =-\frac{64 \pi^{2}}{y^{2}} \partial_{x_{l}} \varphi_{k}\left(\xi_{k}\right)+O\left(\frac{1}{p^{3}}\right)
\end{aligned}
$$

where the last equality is due to the harmonicity of $\varphi_{k}$ near $\xi_{k}$, more precisely,

$$
\frac{1}{2 \pi \varepsilon d\left(\xi_{k}\right)} \int_{\partial B\left(\xi_{k}, \varepsilon d\left(\xi_{k}\right)\right)} \partial_{x_{l}} \varphi_{k}=\partial_{x_{l}} \varphi_{k}\left(\xi_{k}\right),
$$


and by (5.17),

$$
\int_{\partial B\left(\xi_{k}, \varepsilon d\left(\xi_{k}\right)\right)}\left(\partial_{\nu} \varphi_{k} \partial_{x_{l}} \varphi_{k}-\frac{1}{2}\left|\nabla \varphi_{k}\right|^{2} v_{l}\right)=\int_{B\left(\xi_{k}, \varepsilon d\left(\xi_{k}\right)\right)} \Delta \varphi_{k} \partial_{x_{l}} \varphi_{k}=0 .
$$

Accordingly, by (5.16) we get

$$
\partial_{\left(\xi_{k}\right)_{l}} F_{p}^{\lambda}(\xi)=-\frac{64 \pi^{2}}{y^{2}} \partial_{x_{l}} \varphi_{k}\left(\xi_{k}\right)+O\left(\frac{\lambda}{p^{5}}+\frac{1}{p^{3}}\right)=-\frac{32 \pi^{2}}{y^{2}} \partial_{\left(\xi_{k}\right)_{l}} \varphi_{m}^{\lambda}(\xi)+O\left(\frac{\lambda}{p^{5}}\right)+O\left(\frac{1}{p^{3}}\right)
$$

since

$$
\partial_{x_{l}} \varphi_{k}\left(\xi_{k}\right)=\frac{1}{2} \partial_{\left(\xi_{k}\right)_{l}} \varphi_{m}^{\lambda}(\xi)
$$

This completes the proof.

\section{Proof of theorems}

Proof of Theorem 1.1. According to Proposition 5.1, the function $u_{p, \lambda}=U_{\xi}+\phi_{\xi}$, where $U_{\xi}$ is defined in (2.12) and $\phi_{\xi}$ is the unique solution to problem (4.1) given by Proposition 4.1, is a solution to problem (1.1) if we adjust $\xi$ so that it is a critical point of $F_{p}^{\lambda}(\xi)$, defined by (5.3). This is equivalent to finding a critical point of

$$
\widetilde{F}_{p}^{\lambda}(\xi)=-\frac{y^{2}}{32 \pi^{2}}\left[F_{p}^{\lambda}(\xi)-\frac{4 \pi m p}{y^{2}}-\frac{4 \pi m}{y^{2}}-\frac{m}{2 y^{2}} \int_{\mathbb{R}^{2}}\left(\frac{8}{\left(1+|y|^{2}\right)^{2}} U_{1,0}-\Delta \omega_{1}\right)\right] .
$$

On the other hand, from Proposition 5.2 and 5.3, for $\xi=\left(\xi_{1}, \ldots, \xi_{m}\right) \in \mathcal{O}_{\varepsilon}$, we have that

$$
\widetilde{F}_{p}^{\lambda}(\xi)=\varphi_{m}^{\lambda}(\xi)+\frac{m}{2 \pi} \log \lambda+\frac{\log \lambda}{p} \Theta_{p, \lambda}(\xi),
$$

where $\varphi_{m}^{\lambda}$ is given by (1.9), and $\Theta_{p, \lambda}$ and $\nabla_{\xi} \Theta_{p, \lambda}$ are uniformly bounded in the considered region as $p$ and $\lambda$ go to $+\infty$ such that $\frac{\log \lambda}{p}=o(1)$ and $\frac{\lambda}{p^{2} \log \lambda}=O(1)$.

Let us first claim that for any small $\varepsilon>0$, there exists a constant $C>0$ such that

$$
\inf _{\xi \in \partial \mathcal{O}_{\varepsilon}} \widetilde{F}_{p}^{\lambda}(\xi) \geq \frac{m}{2 \pi} \inf _{x \in \partial \Omega} h_{\lambda}\left(\theta_{b(x)}, b(x)\right)+\frac{m}{2 \pi} \log \lambda+\frac{C}{\varepsilon \lambda}-C
$$

uniformly for any large $p$ and $\lambda$ satisfying $\frac{\log \lambda}{p}=o(1)$ and $\lambda p^{2} \log \lambda=O(1)$. To prove this, we recall that

$$
\mathcal{O}_{\varepsilon}=\left\{\xi=\left(\xi_{1}, \ldots, \xi_{m}\right) \in \Omega^{m}:\left|\xi_{j}-\xi_{k}\right| \geq \varepsilon,\left|\lambda d\left(\xi_{j}\right)-\theta_{b\left(\hat{\xi}_{j}\right)}\right| \leq \frac{1}{\varepsilon \lambda^{1 / 2}}, j, k=1, \ldots, m, \dot{l}=k\right\},
$$

where $\varepsilon>0$ is a sufficiently small but fixed number. Obviously, if $\xi=\left(\xi_{1}, \ldots, \xi_{m}\right) \in \partial \mathcal{O}_{\varepsilon}$, then either we have $\left|\lambda d\left(\xi_{j_{0}}\right)-\theta_{b\left(\hat{\xi}_{j_{0}}\right)}\right|=\frac{1}{\varepsilon \lambda^{1 / 2}}$ for some $j_{0}$, or $\left|\xi_{j_{0}}-\xi_{k_{0}}\right|=\varepsilon$ for some $j_{0}=k_{0}$. Using (1.5)-(1.8) and the fact that $h_{\lambda}(\theta, \tilde{b})$ has a unique non-degenerate minimum $\theta_{\tilde{b}} \in(0,+\infty)$ independent of $\lambda$, we have, for $\left|\lambda d\left(\xi_{j_{0}}\right)-\theta_{b\left(\hat{\xi}_{j_{0}}\right)}\right|=\frac{1}{\varepsilon \lambda^{1 / 2}}$, that

$$
\begin{aligned}
\widetilde{F}_{p}^{\lambda}(\xi) & =\sum_{j=1}^{m} H_{\lambda}\left(\xi_{j}, \xi_{j}\right)+\sum_{j=k} G_{\lambda}\left(\xi_{j}, \xi_{k}\right)+\frac{m}{2 \pi} \log \lambda+\frac{\log \lambda}{p} \Theta_{p, \lambda}(\xi) \\
& \geq \frac{1}{2 \pi} h_{\lambda}\left(\theta_{b\left(\hat{\xi}_{j_{0}}\right)} \pm \frac{1}{\varepsilon \lambda^{1 / 2}}, b\left(\hat{\xi}_{j_{0}}\right)\right)+\frac{1}{2 \pi} \sum_{j=j_{0}} h_{\lambda}\left(\theta_{b\left(\hat{\xi}_{j}\right)}, b\left(\hat{\xi}_{j}\right)\right)+\frac{m}{2 \pi} \log \lambda+O\left(\frac{1}{\lambda}\right)+O\left(\frac{1}{p} \log \lambda\right) \\
& \geq \frac{m}{2 \pi} \inf _{x \in \partial \Omega} h_{\lambda}\left(\theta_{b(x)}, b(x)\right)+\frac{m}{2 \pi} \log \lambda+\frac{C}{\varepsilon^{2} \lambda}-C,
\end{aligned}
$$


while for $\left|\xi_{j_{0}}-\xi_{k_{0}}\right|=\varepsilon$, by (3.15),

$$
\begin{aligned}
\widetilde{F}_{p}^{\lambda}(\xi) & =\sum_{j=1}^{m} H_{\lambda}\left(\xi_{j}, \xi_{j}\right)+\sum_{j=k} G_{\lambda}\left(\xi_{j}, \xi_{k}\right)+\frac{m}{2 \pi} \log \lambda+\frac{\log \lambda}{p} \Theta_{p, \lambda}(\xi) \\
& \geq \frac{m}{2 \pi} \inf _{x \in \partial \Omega} h_{\lambda}\left(\theta_{b(x)}, b(x)\right)+G_{\lambda}\left(\xi_{j_{0}}, \xi_{k_{0}}\right)+\frac{m}{2 \pi} \log \lambda+O\left(\frac{1}{\lambda}\right)+O\left(\frac{1}{p} \log \lambda\right) \\
& \geq \frac{m}{2 \pi} \inf _{x \in \partial \Omega} h_{\lambda}\left(\theta_{b(x)}, b(x)\right)+\frac{m}{2 \pi} \log \lambda+\frac{C}{\varepsilon \lambda}-C .
\end{aligned}
$$

We shall show that $\widetilde{F}_{p}^{\lambda}$ has at least two distinct critical points in the region $\mathcal{O}_{\varepsilon}$, a fact that will prove our result. Observe that $\widetilde{F}_{p}^{\lambda}$ is $C^{1}$ and uniformly bounded from below in $\mathcal{O}_{\varepsilon}$, and such that

$$
\inf _{\xi \in \partial \mathcal{O}_{\varepsilon}} \widetilde{F}_{p}^{\lambda}(\xi) \rightarrow+\infty \quad \text { as } \varepsilon \rightarrow 0 .
$$

Hence, since $\varepsilon$ is sufficiently small, $\widetilde{F}_{p}^{\lambda}$ has an absolute minimum $M_{1}^{p, \lambda}$ in $\mathcal{O}_{\varepsilon}$ whenever $p$ and $\lambda$ are sufficiently large such that $\frac{\log \lambda}{p}=o(1)$ and $\frac{\lambda}{p^{2} \log \lambda}=O(1)$. On the other hand, the Lusternik-Schnirelmann theory is applicable in our setting, so that the number of critical points of $\widetilde{F}_{p}^{\lambda}$ in $\mathcal{O}_{\varepsilon}$ can be estimated from below by cat $\mathcal{O}_{\varepsilon}\left(\mathcal{O}_{\varepsilon}\right)$, the Lusternik-Schnirelmann category of $\mathcal{O}_{\varepsilon}$ relative to $\mathcal{O}_{\varepsilon}$. Let us recall that $\operatorname{cat}_{\mathcal{O}_{\varepsilon}}(A)$, the Lusternik-Schnirelmann category of a closed subset $A$ of $\mathcal{O}_{\varepsilon}$ relative to $\mathcal{O}_{\varepsilon}$, is the minimal integer $l$ such that $A$ can be covered by $l$ closed and contractible subsets of $\mathcal{O}_{\varepsilon}$.

We claim that $\operatorname{cat}_{\mathcal{O}_{\varepsilon}}\left(\mathcal{O}_{\varepsilon}\right)>1$. Indeed, by contradiction, assume that cat $\mathcal{O}_{\varepsilon}\left(\mathcal{O}_{\varepsilon}\right)=1$. This means that $\mathcal{O}_{\varepsilon}$ is contractible in itself, namely there exist a point $\xi^{0} \in \mathcal{O}_{\varepsilon}$ and a continuous function $\Gamma:[0,1] \times \mathcal{O}_{\varepsilon} \rightarrow \mathcal{O}_{\varepsilon}$ such that for all $\xi \in \mathcal{O}_{\varepsilon}$,

$$
\Gamma(0, \xi)=\xi \text { and } \Gamma(1, \xi)=\xi^{0} .
$$

Given one connected component $\mathcal{C}_{0}$ of $\partial \Omega$, let $\Lambda: S^{1} \rightarrow \mathcal{C}_{0}$ be a continuous bijective function that parametrizes $\mathcal{C}_{0}$. Observe that for any $x \in \Omega$ close to $\partial \Omega$, there exists a unique point $\hat{x}$ on $\partial \Omega$ closest to $x$. Then, we can define a continuous map $\hat{P}: \mathcal{O}_{\varepsilon} \rightarrow \partial \Omega$ such that $\hat{P} \xi=\hat{\xi}_{1}$ for any $\xi=\left(\xi_{1}, \ldots, \xi_{m}\right) \in \mathcal{O}_{\varepsilon}$. Set

$$
g_{\lambda}(x)=x-\frac{\theta_{b(x)}}{\lambda} v(x), \quad x \in \mathcal{C}_{0},
$$

where $v$ is the exterior unit normal vector of $\partial \Omega$. Obviously, for $\lambda$ sufficiently large, $g_{\lambda}$ is a continuous invertible function. We represent $S^{1}=\{z \in \mathbb{C}:|z|=1\}$. Let $f: S^{1} \rightarrow \mathcal{O}_{\varepsilon}$ be the continuous function defined by

$$
f_{\lambda}(z)=\left(g_{\lambda}(\Lambda(z)), g_{\lambda}\left(\Lambda\left(z e^{i 2 \pi \frac{1}{m}}\right)\right), \ldots, g_{\lambda}\left(\Lambda\left(z e^{i 2 \pi \frac{m-1}{m}}\right)\right)\right), \quad z \in S^{1} .
$$

Let $\eta:[0,1] \times S^{1} \rightarrow S^{1}$ be the well-defined continuous map given by

$$
\eta_{\lambda}(t, z)=\Lambda^{-1} \circ \hat{P} \circ \Gamma\left(t, f_{\lambda}(z)\right) .
$$

The function $\eta_{\lambda}$ is a contraction of $S^{1}$ to a point and this gives a contradiction. Thus, we conclude that $\operatorname{cat}_{\mathcal{O}_{\varepsilon}}\left(\mathcal{O}_{\varepsilon}\right) \geq 2$.

Let us now define

$$
M_{2}^{p, \lambda}=\inf _{A \in \Xi} \sup _{\xi \in A} \widetilde{F}_{p}^{\lambda}(\xi)
$$

where

$$
\Xi=\left\{A \subset \mathcal{O}_{\varepsilon}: A \text { closed and } \operatorname{cat}_{\mathcal{O}_{\varepsilon}}(A) \geq 2\right\} .
$$

By the above construction of $f_{\lambda}$, we have that

$$
\Lambda^{-1} \circ \hat{P} \circ f_{\lambda}(z)=z \quad \text { for any } z \in S^{1},
$$


so $\Lambda^{-1} \circ \hat{P} \circ f_{\lambda}$ has a nonzero winding number, which implies that $\operatorname{cat}_{\mathcal{O}_{\varepsilon}}\left(A_{0}\right) \geq 2$ with $A_{0}=\left\{f_{\lambda}(z): z \in S^{1}\right\}$. Note that for any $\xi=\left(\xi_{1}, \ldots, \xi_{m}\right) \in A_{0}$, the $m$ coordinates of $\xi$ are uniformly separated and independent of $\varepsilon$ and $\lambda$. Then, for any $\xi \in A_{0}$,

$$
\begin{aligned}
\widetilde{F}_{p}^{\lambda}(\xi) & =\sum_{j=1}^{m} H_{\lambda}\left(\xi_{j}, \xi_{j}\right)+\sum_{j=k} G_{\lambda}\left(\xi_{j}, \xi_{k}\right)+\frac{m}{2 \pi} \log \lambda+\frac{\log \lambda}{p} \Theta_{p, \lambda}(\xi) \\
& =\frac{1}{2 \pi} \sum_{j=1}^{m} h_{\lambda}\left(\theta_{b\left(\hat{\xi}_{j}\right)}, b\left(\hat{\xi}_{j}\right)\right)+\frac{m}{2 \pi} \log \lambda+O(1)+O\left(\frac{1}{\lambda}\right)+O\left(\frac{1}{p} \log \lambda\right),
\end{aligned}
$$

so that, using (1.7) and (2.3),

$$
M_{2}^{p, \lambda}=\inf _{A \in \Xi} \sup _{\xi \in A} \widetilde{F}_{p}^{\lambda}(\xi) \leq \sup _{\xi \in A_{0}} \widetilde{F}_{p}^{\lambda}(\xi) \leq C<+\infty
$$

uniformly for any large $p$ and $\lambda$ such that $\frac{\log \lambda}{p}=o(1)$ and $\frac{\lambda}{p^{2} \log \lambda}=O(1)$. Thus, Lusternik-Schnirelmann theory gives that $M_{2}^{p, \lambda}$ is a critical level for $\widetilde{F}_{p}^{\lambda}$.

Obviously, $M_{1}^{p, \lambda} \leq M_{2}^{p, \lambda}$. If $M_{1}^{p, \lambda}<M_{2}^{p, \lambda}$, then we conclude that there are at least two distinct critical points for $\widetilde{F}_{p}^{\lambda}$ (distinct up to permutations) in $\mathcal{O}_{\varepsilon}$ corresponding to two distinct solutions of problem (1.1). If $M_{1}^{p, \lambda}=M_{2}^{p, \lambda}$, then we get that there is at least one set $A$, with cat $_{\mathcal{O}_{\varepsilon}}(A) \geq 2$, where the function $\widetilde{F}_{p}^{\lambda}$ reaches its absolute minimum. In this case, we conclude that there are infinitely many critical points for $\widetilde{F}_{p}^{\lambda}$ in $\mathcal{O}_{\varepsilon}$. Since permutations are only finite in number, the result is thus proven.

Proof of Theorem 1.2. For the case $m=1$, it suffices to prove the existence of critical points of $\widetilde{F}_{p}^{\lambda}$ given by (6.1), whose proof is based on the asymptotic formulas (1.5)-(1.6) for $H_{\lambda}(\xi, \xi)$ combined with a linking argument.

In fact, for $m=1$, by (6.1) for any large $p$ and $\lambda$ satisfying $\frac{1}{p} \log \lambda=o(1)$, we get that

$$
\widetilde{F}_{p}^{\lambda}(\xi)=H_{\lambda}(\xi, \xi)+\frac{1}{2 \pi} \log \lambda+\frac{\log \lambda}{p} \Theta_{p, \lambda}(\xi), \quad \xi \in \mathcal{O}_{\varepsilon},
$$

where

$$
\frac{\log \lambda}{p}\left|\Theta_{p, \lambda}(\xi)\right|=O\left(\frac{\log \lambda}{p}\right)=o(1), \quad \frac{\log \lambda}{p}\left|\nabla_{\xi} \Theta_{p, \lambda}(\xi)\right|=O\left(\frac{\lambda}{p^{3}}\right)+O\left(\frac{1}{p}\right)
$$

and

$$
\mathcal{O}_{\varepsilon}=\left\{\xi \in \Omega:\left|\lambda d(\xi)-\theta_{b(\hat{\xi})}\right| \leq \frac{1}{\varepsilon \lambda^{1 / 2}}\right\} .
$$

Using the asymptotic formulas of $H_{\lambda}(\xi, \xi)$ in (1.5)-(1.6), for $\xi \in \mathcal{O}_{\varepsilon}$, we have that

$$
\widetilde{F}_{p}^{\lambda}(\xi)=\frac{1}{2 \pi} h_{\lambda}(\lambda d(\xi), b(\hat{\xi}))+\frac{1}{2 \pi} \log \lambda+O\left(\frac{1}{\lambda}\right)+O\left(\frac{1}{p} \log \lambda\right) .
$$

Note that for any $\tilde{b}>0$ the function $h_{\lambda}(\cdot, \tilde{b})$ has a unique non-degenerate minimum $\theta_{\tilde{b}} \in(0,+\infty)$, which is a continuous function with respect to the variable $\tilde{b}$. We define

$$
S^{\star}:=\left\{\xi \in \Omega: \lambda d(\xi)=\theta_{b(\hat{\xi})}\right\} \subset \mathcal{O}_{\varepsilon} .
$$

Then, for $\varepsilon>0$ sufficiently small, it follows that

$$
\inf _{\xi \in \partial \mathcal{O}_{\varepsilon}} h_{\lambda}(\lambda d(\xi), b(\hat{\xi}))>\sup _{\xi \in S^{\star}} h_{\lambda}(\lambda d(\xi), b(\hat{\xi})),
$$

and therefore, by (6.5),

$$
\inf _{\xi \in \partial \mathcal{O}_{\varepsilon}} \widetilde{F}_{p}^{\lambda}(\xi)>\sup _{\xi \in S^{\star}} \widetilde{F}_{p}^{\lambda}(\xi)
$$

uniformly for any large $p$ and $\lambda$ satisfying $\frac{1}{p} \log \lambda=o(1)$. Accordingly, there exists $\xi_{1, p, \lambda} \in \mathcal{O}_{\varepsilon}$ such that

$$
\inf _{\xi \in \mathcal{O}_{\varepsilon}} \widetilde{F}_{p}^{\lambda}(\xi)=\widetilde{F}_{p}^{\lambda}\left(\xi_{1, p, \lambda}\right) .
$$


We now seek another critical point of $\widetilde{F}_{p}^{\lambda}$ in $\mathcal{O}_{\varepsilon}$. For each $x \in \partial \Omega$, we consider the set

$$
Q_{x}:=\left\{\xi \in \mathcal{O}_{\varepsilon}: \hat{\xi}=x\right\} .
$$

Let us assume that there exists $x_{0} \in \partial \Omega$ such that

$$
\inf _{\xi \in Q_{x_{0}}} \widetilde{F}_{p}^{\lambda}(\xi)>\widetilde{F}_{p}^{\lambda}\left(\xi_{1, p, \lambda}\right) .
$$

Otherwise, if such a point does not exist then the theorem is proved. Let $\partial Q_{x_{0}}$ denote the boundary of $Q_{x_{0}}$. Obviously, the sets $\partial Q_{x_{0}}$ and $S^{\star}$ link in $\mathcal{O}_{\varepsilon}$. Moreover, by (6.6) we have

$$
\inf _{\xi \in \partial Q_{x_{0}}} \widetilde{F}_{p}^{\lambda}(\xi)>\sup _{\xi \in S^{*}} \widetilde{F}_{p}^{\lambda}(\xi)
$$

Let

$$
\mathcal{P}=\left\{f \in C^{0}\left(\bar{Q}_{x_{0}}, \overline{\mathcal{O}}_{\varepsilon}\right):\left.f\right|_{\partial Q_{x_{0}}}=\left.\operatorname{Id}\right|_{\partial Q_{x_{0}}}\right\}
$$

Then,

$$
\beta=\sup _{f \in \mathcal{P}} \inf _{\xi \in Q_{x_{0}}} \widetilde{F}_{p}^{\lambda}(\xi)
$$

is a critical value of $\widetilde{F}_{p}^{\lambda}$ which is different from $\widetilde{F}_{p}^{\lambda}\left(\xi_{1, p, \lambda}\right)$ by (6.7). Hence, we obtain the existence of the second critical point $\xi_{2, p, \lambda}$ for $\widetilde{F}_{p}^{\lambda}$ in $\mathcal{O}_{\varepsilon}$, which is different from $\xi_{1, p, \lambda}$.

Proof of Theorem 1.3. Let $x_{0} \in \partial \Omega$ be a non-degenerate critical point of the mean curvature $\kappa$ and $b \equiv 1$. As in the proof of Theorem 1.2, it suffices to prove the existence of critical points of $\widetilde{F}_{p}^{\lambda}$ near $x_{0}$, where $\widetilde{F}_{p}^{\lambda}$ is given by (6.2)-(6.4). For this purpose, we will take advantage of the asymptotic formula (1.6) of $H_{\lambda}(\xi, \xi)$ to relate the topological degree of the $\nabla \widetilde{F}_{p}^{\lambda}$ in a suitable small set close to $x_{0}$ with that of the $\nabla \kappa$.

Let $R_{\lambda}(\xi)=H_{\lambda}(\xi, \xi)$ and $\nabla_{\mathrm{T}}$ denote the tangential gradient which is defined in a neighborhood of $\partial \Omega$. For any $\alpha \in(0,1)$, there exist the following expansions for the gradient of $R_{\lambda}(\xi)$ (see [7]):

$$
\begin{gathered}
\nabla_{\mathrm{T}} R_{\lambda}(\xi)=\frac{1}{2 \pi \lambda} \nabla \kappa(\hat{\xi}) \mathrm{v}(\lambda d(\xi))+O\left(\frac{1}{\lambda^{1+\alpha}}\right), \\
\left\langle\nabla R_{\lambda}(\xi), v(\hat{\xi})\right\rangle=-\frac{\lambda}{2 \pi} \frac{\partial h_{\lambda}}{\partial \theta}(\lambda d(\xi), 1)-\frac{1}{2 \pi} \kappa(\hat{\xi}) \mathrm{v}^{\prime}(\lambda d(\xi))+O\left(\frac{1}{\lambda^{\alpha}}\right)
\end{gathered}
$$

uniformly for $\left|\lambda d(\xi)-\theta_{b}\right| \leq \frac{1}{\varepsilon \lambda^{1 / 2}}$ with $b \equiv 1$.

Since $x_{0} \in \partial \Omega$ is a non-degenerate critical point of $\kappa$, there exist $C>0$ and $\sigma>0$ such that

$$
|\nabla \kappa(\hat{\xi})| \geq C\left|\hat{\xi}-x_{0}\right| \text { for all } \hat{\xi} \in \partial \Omega \text { such that }\left|\hat{\xi}-x_{0}\right| \leq \sigma .
$$

On the other hand, we know that the function $h_{\lambda}(\cdot, 1)$ has a unique minimum $\theta_{b}>0$, which is nondegenerate, and hence by taking $C>0$ and $\sigma>0$ smaller if necessary, we have

$$
\left|\frac{\partial}{\partial \theta} h_{\lambda}(\theta, 1)\right| \geq C\left|\theta-\theta_{b}\right| \quad \text { for all }\left|\theta-\theta_{b}\right| \leq \sigma .
$$

Using the fact that the function $\mathrm{v}$ is continuous and negative in $\mathbb{R}$, we can choose $\sigma>0$ smaller such that

$$
\inf _{\theta \in\left[\theta_{b}-\sigma, \theta_{b}+\sigma\right]}|\mathrm{v}(\theta)|>0 .
$$

Let $0<\beta<\alpha$. For $\lambda$ large and $\varepsilon$ small but fixed, we can also assume $\max \left\{\frac{1}{\varepsilon \lambda^{1 / 2}}, \frac{1}{\lambda^{\beta}}\right\}<\sigma<\theta_{b}$, since $\theta_{b}>0$. Consider the compact set

$$
\mathcal{K}_{\lambda}:=\left\{\xi \in \Omega:\left|\lambda d(\xi)-\theta_{b}\right| \leq \frac{1}{\varepsilon \lambda^{1 / 2}},\left|\hat{\xi}-x_{0}\right| \leq \frac{1}{\lambda^{\beta}}\right\} .
$$

Then, $\mathcal{K}_{\lambda} \subset \mathcal{O}_{\varepsilon}$ with $\mathcal{O}_{\varepsilon}$ defined in (6.4). Let

$$
\widetilde{F}_{\lambda}^{0}(\xi)=\frac{1}{2 \pi} h_{\lambda}(\lambda d(\xi), 1)+\frac{1}{2 \pi \lambda} \kappa(\hat{\xi}) \mathrm{v}(\lambda d(\xi))
$$


Obviously, $\widetilde{F}_{\lambda}^{0}$ has a unique non-degenerate critical point in the interior of $\mathcal{K}_{\lambda}$. Now we define the homotopy between $\widetilde{F}_{\lambda}^{p}$ and $\widetilde{F}_{\lambda}^{0}$ as follows:

$$
\widetilde{F}_{\lambda}^{p, t}(\xi)=t \widetilde{F}_{\lambda}^{p}(\xi)+(1-t) \widetilde{F}_{\lambda}^{0}(\xi), \quad t \in[0,1] .
$$

We observe that for any large $p$ and $\lambda$ satisfying $\frac{1}{p} \log \lambda=o(1)$, there exists $C^{\prime}>0$ such that if $\xi \in \partial \mathcal{K}_{\lambda}$ then: 1. if $\left|\lambda d(\xi)-\theta_{b}\right|=\frac{1}{\varepsilon \lambda^{1 / 2}}$, then by (6.2), (6.3), (6.9) and (6.11), we have

$$
\left|\nabla \widetilde{F}_{\lambda}^{p, t}(\xi)\right| \geq\left|\left\langle\nabla \widetilde{F}_{\lambda}^{p, t}(\xi), v(\hat{\xi})\right\rangle\right| \geq C^{\prime} \sqrt{\lambda}+O\left(\frac{\lambda}{p^{3}}\right)+O\left(\frac{1}{p}\right) ;
$$

2. if $\left|\hat{\xi}-x_{0}\right|=\frac{1}{\lambda^{\beta}}$, then by (6.2), (6.3), (6.8), (6.10) and (6.12), we have

$$
\left|\nabla \widetilde{F}_{\lambda}^{p, t}(\xi)\right| \geq\left|\nabla_{\mathrm{T}} \widetilde{F}_{\lambda}^{p, t}(\xi)\right| \geq \frac{C^{\prime}}{\lambda^{1+\beta}}+O\left(\frac{\lambda}{p^{3}}\right)+O\left(\frac{1}{p}\right) .
$$

Then, it is easily checked that by choosing sufficiently large $p$ and $\lambda$ such that $\frac{1}{p} \lambda^{1+\beta}=o(1),\left|\nabla \widetilde{F}_{\lambda}^{p, t}(\xi)\right|$ remains uniformly positive on $\partial \mathcal{K}_{\lambda}$. By the degree theory $\widetilde{F}_{\lambda}^{p}(\xi)=\widetilde{F}_{\lambda}^{p, 1}(\xi)$ has a critical point in the interior of $\mathcal{K}_{\lambda}$, and hence it is located at distance $O\left(\lambda^{-\beta}\right)$ from $x_{0}$.

Proof of Theorem 1.4. Let $x_{0} \in \partial \Omega$ be a non-degenerate critical point of the function $b$. The proof is similar to that of Theorem 1.3, that is, to prove the existence of critical points of $\widetilde{F}_{p}^{\lambda}$ defined in (6.2)-(6.4), we will take advantage of the asymptotic formula (1.5) of $H_{\lambda}(\xi, \xi)$ to relate the topological degree of the $\nabla \widetilde{F}_{p}^{\lambda}$ in a suitable small set close to $x_{0}$ with that of the $\nabla b$.

Note that from [7], the gradient of $R_{\lambda}(\xi)$ has the following expansions:

$$
\begin{aligned}
\nabla_{\mathrm{T}} R_{\lambda}(\xi) & =\frac{1}{2 \pi} \frac{\partial h_{\lambda}}{\partial \tilde{b}}(\lambda d(\xi), b(\hat{\xi})) \nabla b(\hat{\xi})+O\left(\frac{1}{\lambda}\right), \\
\left\langle\nabla R_{\lambda}(\xi), v(\hat{\xi})\right\rangle & =-\frac{\lambda}{2 \pi} \frac{\partial h_{\lambda}}{\partial \theta}(\lambda d(\xi), b(\hat{\xi}))+O(1)
\end{aligned}
$$

uniformly for $\left|\lambda d(\xi)-\theta_{b(\hat{\xi})}\right| \leq \frac{1}{\varepsilon \lambda^{1 / 2}}$.

Since $x_{0} \in \partial \Omega$ is a non-degenerate critical point of the function $b$, there exist $C>0$ and $\sigma>0$ such that

$$
|\nabla b(\hat{\xi})| \geq C\left|\hat{\xi}-x_{0}\right| \quad \text { for all } \hat{\xi} \in \partial \Omega \text { such that }\left|\hat{\xi}-x_{0}\right| \leq \sigma .
$$

On the other hand, we know that for any $\tilde{b}>0$ the function $h_{\lambda}(\cdot, \tilde{b})$ has a unique non-degenerate minimum $\theta_{\tilde{b}} \in(0,+\infty)$, which is a continuous function with respect to the variable $\tilde{b}$, and hence taking $C>0$ and $\sigma>0$ smaller if necessary, we have

$$
\left|\frac{\partial}{\partial \theta} h_{\lambda}(\theta, b(\hat{\xi}))\right| \geq C\left|\theta-\theta_{b(\hat{\xi})}\right| \quad \text { for all }\left|\theta-\theta_{b(\hat{\xi})}\right| \leq \sigma \text { and } \hat{\xi} \in \partial \Omega .
$$

Using that the function $\frac{\partial h_{\lambda}}{\partial \tilde{b}}(\theta, \tilde{b})$ is continuous and negative in $\mathbb{R}_{+} \times \mathbb{R}_{+}$, we can choose $\sigma>0$ smaller such that

$$
\inf _{\hat{\xi} \in \partial \Omega, \theta \in\left[\theta_{b(\hat{\xi})^{-\sigma}}\right.}\left|\frac{\partial}{\left.b_{b(\xi)}+\sigma\right]}\right| \frac{\partial}{\partial \tilde{b}} h_{\lambda}(\theta, b(\hat{\xi})) \mid>0 .
$$

Let $0<\beta<1$. For large $\lambda$ and small but fixed $\varepsilon$, we can also assume $\max \left\{\frac{1}{\varepsilon \lambda^{1 / 2}}, \frac{1}{\lambda^{\beta}}\right\}<\sigma<\min _{\hat{\xi} \in \partial \Omega} \theta_{b(\hat{\xi})}$, since the function $\theta_{b(\cdot)}$ is continuous and positive on $\partial \Omega$. Consider the compact set

$$
\mathcal{K}_{\lambda}:=\left\{\xi \in \Omega:\left|\lambda d(\xi)-\theta_{b(\hat{\xi})}\right| \leq \frac{1}{\varepsilon \lambda^{1 / 2}},\left|\hat{\xi}-x_{0}\right| \leq \frac{1}{\lambda^{\beta}}\right\} .
$$

Then, $\mathcal{K}_{\lambda} \subset \mathcal{O}_{\varepsilon}$ with $\mathcal{O}_{\varepsilon}$ defined in (6.4). Let

$$
\widetilde{F}_{\lambda}^{0}(\xi)=\frac{1}{2 \pi} h_{\lambda}(\lambda d(\xi), b(\hat{\xi}))
$$


Obviously, $\widetilde{F}_{\lambda}^{0}$ has a unique non-degenerate critical point $\xi_{\lambda}$ located in the interior of $\mathcal{K}_{\lambda}$ such that $\hat{\xi}_{\lambda}=x_{0}$ and $\lambda d\left(\xi_{\lambda}\right)=\theta_{b\left(x_{0}\right)}$. Now we define the homotopy between $\widetilde{F}_{\lambda}^{p}$ and $\widetilde{F}_{\lambda}^{0}$ as follows:

$$
\widetilde{F}_{\lambda}^{p, t}(\xi)=t \widetilde{F}_{\lambda}^{p}(\xi)+(1-t) \widetilde{F}_{\lambda}^{0}(\xi), \quad t \in[0,1] .
$$

We observe that for any large $p$ and $\lambda$ satisfying $\frac{1}{p} \log \lambda=o(1)$, there exists $C^{\prime}>0$ such that if $\xi \in \partial \mathcal{K}_{\lambda}$ then: 1. if $\left|\lambda d(\xi)-\theta_{b(\hat{\xi})}\right|=\frac{1}{\varepsilon \lambda^{1 / 2}}$, then by (6.2), (6.3), (6.14) and (6.16), we have

$$
\left|\nabla \widetilde{F}_{\lambda}^{p, t}(\xi)\right| \geq\left|\left\langle\nabla \widetilde{F}_{\lambda}^{p, t}(\xi), v(\hat{\xi})\right\rangle\right| \geq C^{\prime} \sqrt{\lambda}+O\left(\frac{\lambda}{p^{3}}\right)+O\left(\frac{1}{p}\right) ;
$$

2. $\quad$ if $\left|\hat{\xi}-x_{0}\right|=\frac{1}{\lambda^{\beta}}$, then by (6.2), (6.3), (6.13), (6.15) and (6.17), we have

$$
\left|\nabla \widetilde{F}_{\lambda}^{p, t}(\xi)\right| \geq\left|\nabla_{\mathrm{T}} \widetilde{F}_{\lambda}^{p, t}(\xi)\right| \geq \frac{C^{\prime}}{\lambda^{\beta}}+O\left(\frac{1}{\lambda}\right)+O\left(\frac{\lambda}{p^{3}}\right)+O\left(\frac{1}{p}\right) .
$$

Then, it is easily checked that, choosing sufficiently large $p$ and $\lambda$ such that $\frac{1}{p}\left(\lambda^{1 / 3(1+\beta)}+\lambda^{\beta}\right)=o(1),\left|\nabla \widetilde{F}_{\lambda}^{p, t}(\xi)\right|$ remains uniformly positive on $\partial \mathcal{K}_{\lambda}$. By the degree theory, $\widetilde{F}_{\lambda}^{p}(\xi)=\widetilde{F}_{\lambda}^{p, 1}(\xi)$ has a critical point in the interior of $\mathcal{K}_{\lambda}$, and hence it is located at distance $O\left(\lambda^{-\beta}\right)$ from $x_{0}$.

\section{References}

[1] Adimurthi and M. Grossi, Asymptotic estimates for a two-dimensional problem with polynomial nonlinearity, Proc. Amer. Math. Soc. 132 2004, 1013-1019.

[2] S. Baraket and F. Pacard, Construction of singular limits for a semilinear elliptic equation in dimension 2, Calc. Var. Partial Differential Equations 6 1998, 1-38.

[3] H. Berestycki and J. Wei, On singular perturbation problems with Robin boundary condition, Ann. Sc. Norm. Super. Pisa Cl. Sci. (5) 2 2003, 199-230.

[4] D. Chae and O. Imanuvilov, The existence of non-topological multivortex solutions in the relativistic self-dual ChernSimons theory, Comm. Math. Phys. 215 2000, 119-142.

[5] W. Chen and C. Li, Classification of solutions of some nonlinear elliptic equations, Duke Math. J. 63 1991, 615-622.

[6] D. Daners, Robin boundary value problems on arbitrary domains, Trans. Amer. Math. Soc. 352 2000, 4207-4236.

[7] J. Dávila, M. Kowalczyk and M. Montenegro, Critical points of the regular part of the harmonic Green function with Robin boundary condition, J. Funct. Anal. 255 2008, 1057-1101.

[8] J. Dávila and E. Topp, Concentrating solutions of the Liouville equation with Robin boundary condition, J. Differential Equations 252 2012, 2648-2697.

[9] M. del Pino, M. Kowalczyk and M. Musso, Singular limits in Liouville-type equations, Calc. Var. Partial Differential Equations 24 2005, 47-81.

[10] R. Dillon, P. K. Maini and H. G. Othmer, Pattern formation in generalized Turing systems. I: Steady-state patterns in systems with mixed boundary conditions, J. Math. Biol. 32 1994, 345-393.

[11] K. El Mehdi and M. Grossi, Asymptotic estimates and qualitative properties of an elliptic problem in dimension two, Adv. Nonlinear Stud. 4 2004, 15-36.

[12] P. Esposito, M. Grossi and A. Pistoia, On the existence of blowing-up solutions for a mean field equation, Ann. Inst. H. Poincaré Anal. Non Linéaire 22 2005, 227-257.

[13] P. Esposito, M. Musso and A. Pistoia, Concentrating solutions for a planar elliptic problem involving nonlinearities with large exponent, J. Differential Equations 227 2006, 29-68.

[14] D. Gilbarg and N. S. Trudinger, Elliptic Partial Differential Equations of Second Order, Springer, Berlin, 2001.

[15] M. Grossi and F. Takahashi, Nonexistence of multi-bubbles solutions to some elliptic equations on convex domains, J. Funct. Anal. 259 2010, 904-917.

[16] W.-M. Ni, Diffusion, cross-diffusion, and their spike-layer steady states, Notices Amer. Math. Soc. 45 1998, 9-18.

[17] W.-M. Ni and I. Takagi, On the shape of least-energy solutions to a semilinear Neumann problem, Comm. Pure Appl. Math. 44 1991, 819-851.

[18] W.-M. Ni and I. Takagi, Locating the peaks of least-energy solutions to a semilinear Neumann problem, Duke Math. J. 70 1993, 247-281.

[19] W.-M. Ni and J. Wei, On the location and profile of spike-layer solutions to singularly perturbed semilinear Dirichlet problems, Comm. Pure Appl. Math. 48 1995, 731-768. 
[20] X. Ren and J. Wei, On a two-dimensional elliptic problem with large exponent in nonlinearity, Trans. Amer. Math. Soc. 343 1994, 749-763.

[21] X. Ren and J. Wei, Single-point condensation and least-energy solutions, Proc. Amer. Math. Soc. 124 1996, 111-120. 